\title{
Herbal medicine derived carbon dots: synthesis and applications in therapeutics, bioimaging and sensing
}

\author{
Wei-Kang Luo' ${ }^{1}$, Liang-Lin Zhang ${ }^{1}$, Zhao-Yu Yang ${ }^{1}$, Xiao-Hang Guo ${ }^{2}$, Yao Wu' ${ }^{1}$, Wei Zhang ${ }^{3}$, Jie-Kun Luo', \\ Tao Tang ${ }^{1}$ and Yang Wang ${ }^{1 *}$ (D)
}

\begin{abstract}
Since the number of raw material selections for the synthesis of carbon dots (CDs) has grown extensively, herbal medicine as a precursor receives an increasing amount of attention. Compared with other biomass precursors, CDs derived from herbal medicine (HM-CDs) have become the most recent incomer in the family of CDs. In recent ten years, a great many studies have revealed that HM-CDs tend to be good at theranostics without drug loading. However, the relevant development and research results are not systematically reviewed. Herein, the origin and history of HM-CDs are outlined, especially their functional performances in medical diagnosis and treatment. Besides, we sort out the herbal medicine precursors, and analyze the primary synthetic methods and the key characteristics. In terms of the applications of $\mathrm{HM}-\mathrm{CD}$, medical therapeutics, ion and molecular detection, bioimaging, as well as pH sensing are summarized. Finally, we discuss the crucial challenges and future prospects.
\end{abstract}

Keywords: Herbal medicine, Carbon dots, Synthesis, Theranostics, Medical applications, Nanomedicine

\section{Introduction}

Initially discovered by Scrivens in early 2004, carbon dots (CDs) are emerging as a novel nanomaterial which is smaller than $10 \mathrm{~nm}$ in size [1]. CDs not only possess the merits from traditional semiconductors (inorganic quantum dots, etc.) and small molecules (fluorophores, etc.) but also exhibit unique properties such as photobleaching resistance, photostability, good biocompatibility and stable physicochemical characteristics [2-5]. These strengths attract much attention in various fields [6-11], especially biomedical theranostics which integrate diagnostics with therapeutics [12].

Many chemical materials (including citric acid [13], ethylene glycol [13], phenol/formaldehyde resins [14],

\footnotetext{
*Correspondence: wangyang_xy87@csu.edu.cn

${ }^{1}$ Institute of Integrative Medicine, Department of Integrated Chinese

and Western Medicine, Xiangya Hospital Central South University, Changsha, China

Full list of author information is available at the end of the article
}

$\mathrm{ZnO}$ [15], poly ethylene imine [16] and other organic solvents) are the primary precursors for CDs creation [17]. Unfortunately, these chemical-based syntheses typically involve toxic products (such as oxidative stress, reactive oxygen species (ROS), inflammation and release of metal ion) [18], that hampers clinical applications $[19,20]$. To meet the challenges, scientists are devoted to searching for biomass-sourced precursors as the replaceable candidates because of their low toxicity, abundant heteroatoms, and good biocompatibility [21]. Many CDs derived from green precursors have been reviewed previously [20, 22, 23]. These reported functional greenCDs are mainly administered for anti-tumor therapy, and some were used as antimicrobial agents [5, 24-27]. Although green-CDs move a steady step on the prospective way towards biomedicine, they cannot eliminate the disadvantages of drug loading (complex manipulation and uncontrollable loading efficiency) [20]. original author(s) and the source, provide a link to the Creative Commons licence, and indicate if changes were made. The images or other third party material in this article are included in the article's Creative Commons licence, unless indicated otherwise in a credit line to the material. If material is not included in the article's Creative Commons licence and your intended use is not permitted by statutory regulation or exceeds the permitted use, you will need to obtain permission directly from the copyright holder. To view a copy of this licence, visit http://creativecommons.org/licenses/by/4.0/. The Creative Commons Public Domain Dedication waiver (http://creativeco mmons.org/publicdomain/zero/1.0/) applies to the data made available in this article, unless otherwise stated in a credit line to the data. 
To achieve low toxicity and avoid delivery cargoes, researchers are turning their attention to the green precursors with specific efficacy [20, 28]. Among these green precursors, herbal medicine is selected as the ideal choice. Because medicinal herbs are the natural products with huge output and approximately innocuousness. Additionally, the unique mode of diagnosis and treatment makes herbal medicine plays a key role in treating diseases [29-31]. More importantly, herbal medicine is rich in active components, thus has multiple pharmacodynamic substances [32]. (Fig. 1) These features induce herbal medicine to become a direct avenue for acquiring heteroatoms, pushing the realization of theranostics in the absence of complex delivery carries. Therefore, CDs derived from herbal medicine (HM-CDs) have attracted intensive attention in the last few years. However, no systematic discussion on the general knowledge of HM-CDs is available so far.

In this review, we aim to highlight the merits and importance of HM-CDs. Four major sections are focused upon: (1) brief history of HM-CDs; (2) herbal medicine precursors; (3) synthetic methods; and (4) applications in biomedicine (diseases and mechanisms), ion and molecule detection, bioimaging, as well as $\mathrm{pH}$ sensing. Finally, we conclude with a discussion of the challenges and perspectives. The scheme of this review is clearly displayed in Fig. 2.

\section{A brief history of HM-CDs}

As early as 2012, Zhou et al. [33] synthesized a water-soluble fluorescent $\mathrm{CD}$ using watermelon peel that is a waste and reproducible raw resource. These CDs showed strong blue luminescence without chemical oxidation and surface passivations, and they were applied in the HeLa cell imaging (Fig. 3). As an herbal medicine, watermelon peel improves fasting blood glucose and changes in hepatic metabolite accumulation [34]. The CDs derived from watermelon peel were not only the first invented HMCDs, but also the first HM-CDs for live cell imaging. This work pioneered HM-CDs as high-performance optical imaging probes.

In 2014, Li et al. [28] obtained the CDs prepared from ginger which is the first HM-CDs used for cell intervention and disease treatment. They found that ginger CDs without drug loading effectively inhibit human hepatocellular carcinoma cells and slower tumor growth in nude mice [28] (Fig. 4). Since then, the synthesis of CDs has initiated an age of herbal medicine-based disease treatment.

Practical compatibility is a key distinctive feature of herbal medicine [35, 36]. The effects of prescription are multi-components, multi-targets, multi-pathways and co-regulatory characters [37]. Jiaosanxian (JSX), a combination of Fructus Crataegi (Jiaoshanzha), Fructus Hordei Germinatus (Jiaomaiya) and Massa Medicata

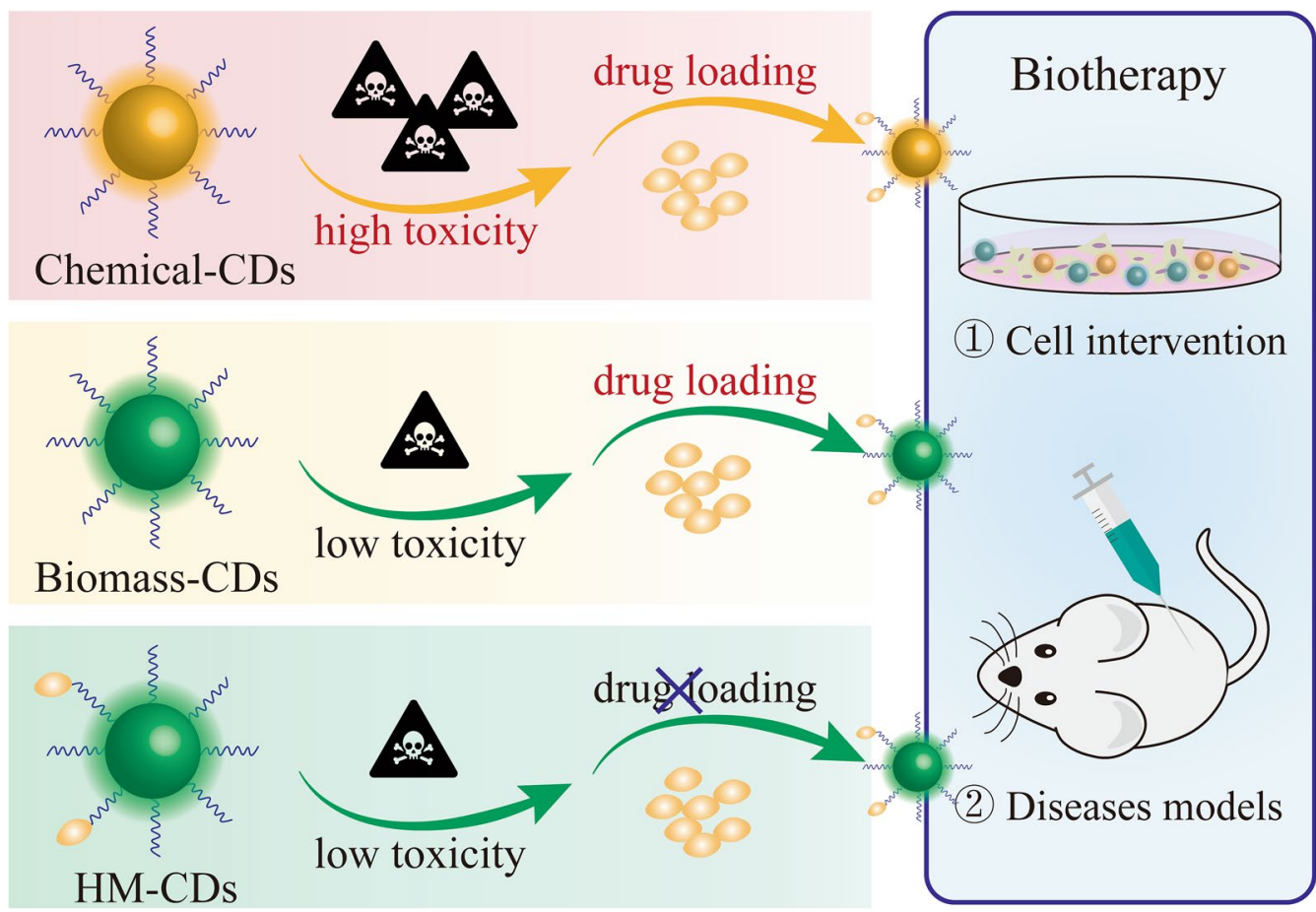

Fig. 1 Schematic diagram of comparison of chemical-CDs, biomass-CDs and HM-CDs in biotherapy 


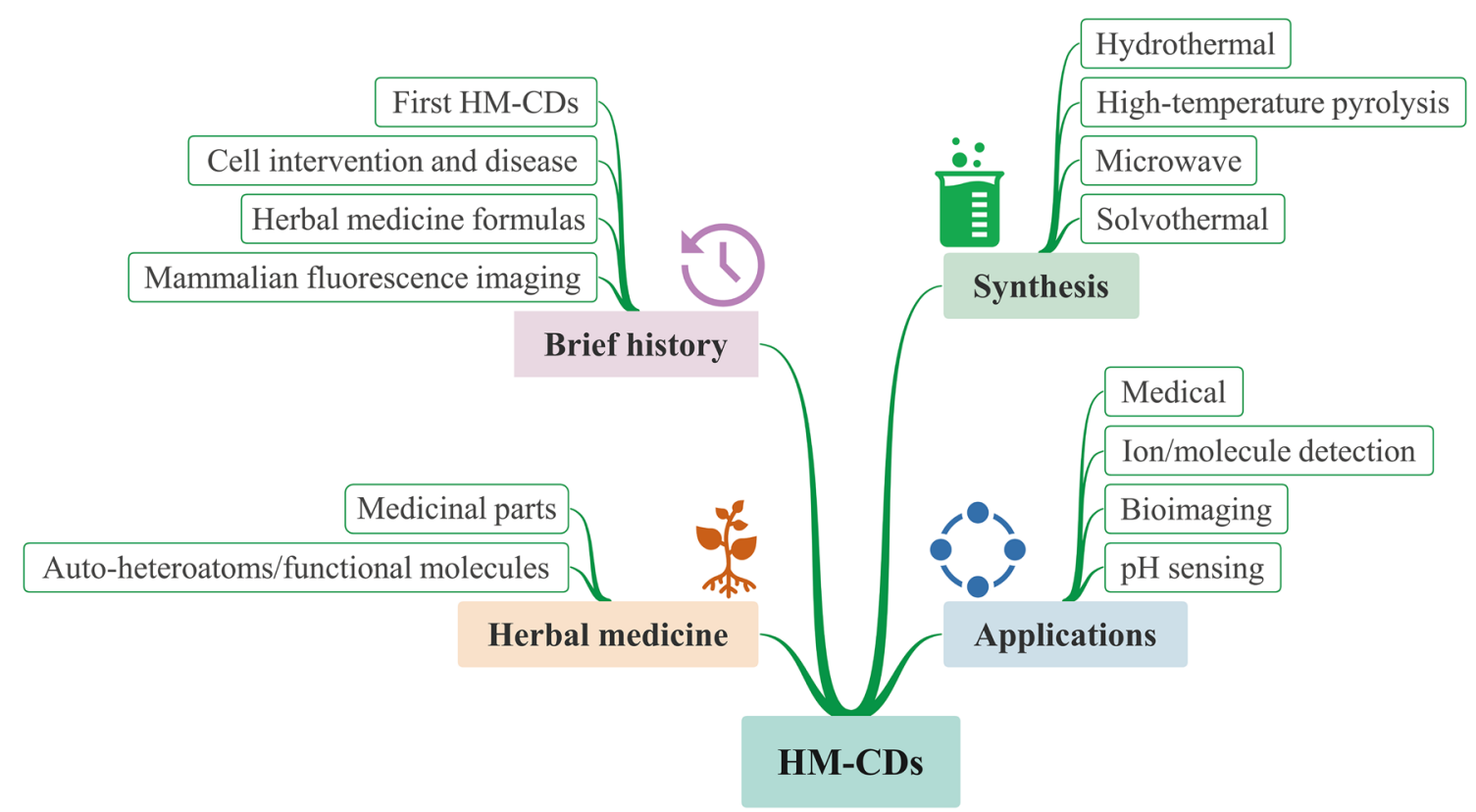

Fig. 2 Schematic diagram of the review
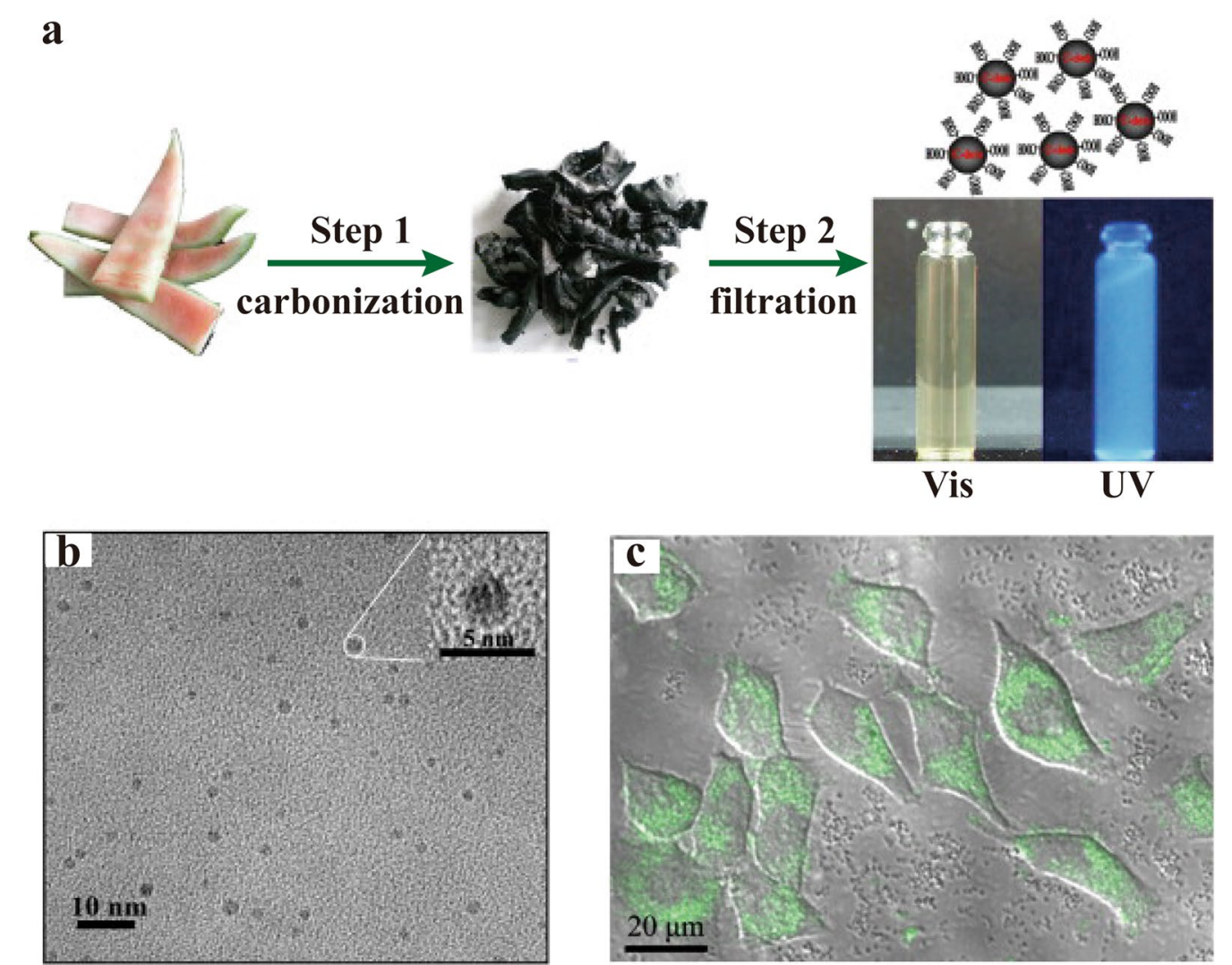

Fig. 3 CDs derived from watermelon peels. a The synthesis procedure. $\mathbf{b}$ HRTEM image and the higher magnification image (inset). c Confocal microscopy image of Hela cells incubated with watermelon peel-CDs. Reprinted with permission from ref. [33]. Copyright (2012) Elsevier 


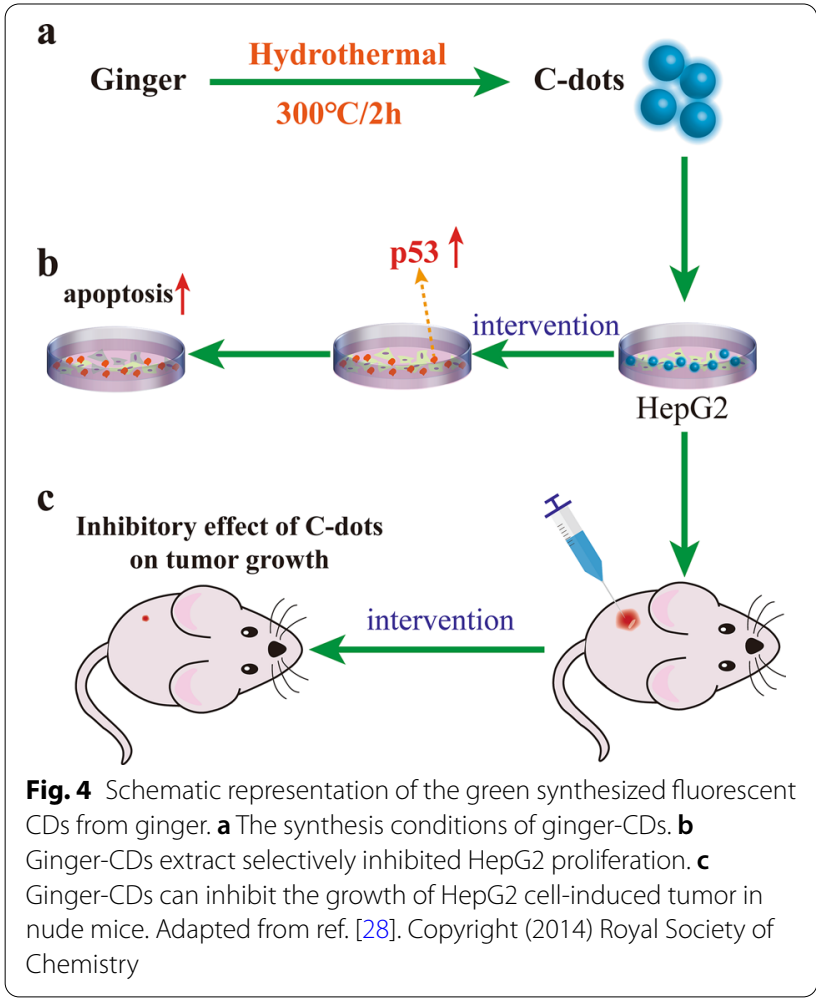

Fermentata (Jiaoshenqu), was the first herbal medicine formula for synthesizing HM-CDs. Thereupon, JSX-CDs laid milestones in the CDs derived from herbal medicine formulas. Regrettably, while this cocktail therapy is in line with the model of clinical applications of herbal medicine, JSX-CDs have more complex surface compositions and structures. Other herbal prescriptions are not used as carbon precursors so far.

CDs derived from Panax notoginseng (Sanqi) for mammalian fluorescence imaging were not found until 2020 [38]. Compared with live cell imaging, in vivo live imaging tends to a clinical transformation. As of today, tens of herbal medicines have been successfully used as precursors of HM-CDs synthesis (Fig. 5).

\section{Herbal medicine for HM-CDs synthesis Medicinal parts}

After analysis, all herbal precursors, we find that the overwhelming majority of all reported herbal precursors are medicinal plants including roots, flowers, leaves, fruits, seeds and other parts (Fig. 6). In herbal medicine systems, plants are also the primary therapeutic agents for treating diseases [32]. Remarkably, different medicinal parts generally contain various active components [39-41]. Since there is no detailed report on the active ingredients of $\mathrm{HM}-\mathrm{CDs}$, we are unable to elaborate on the potential relationship between HM-CD derived from different medicinal parts and their source ingredients. In addition, seldom medicines from hair [42], honey [43], egg yolk oil [44] and mulberry silkworm cocoon [45] are also used for precursors (Table 1). Future studies are urgent to explore a broader range of herbal medicine and not limited to plants.

\section{Auto-heteroatoms and functional molecules}

Due to the diversity and complexity, the structures and photoluminescence mechanisms of CDs, remain poorly understood [46]. Several hypotheses have been proposed to explain the photoluminescence, such as electronic bandgap transitions of conjugated $\pi$-domains, size-dependent emission (quantum confinement effect), surface state-derived luminescence (e.g., surface defects, functional groups) and molecular luminophores, etc. [47-50]. The surface state is considered to be the primary factor [4]. Heteroatom doping (mainly $\mathrm{N}$ dopping [51], S dopping [52], B dopping [53], metal dopping [54]) becomes an effective way to adjust the fluorescent properties of CDs $[55,56]$. The synergistic effects between various heteroatoms lead to more surface defects, reduce nonradiative recombination, thus improve their fluorescence intensity (e.g., B, S, N doped-CDs [57], N, P dopedCDs [58] and N, S doped-CDs [59]). Briefly speaking, a growing appreciation that precursors with abundant heteroatoms may avoid the additional doping.

As we all know, natural sources consist of organic molecules which tend to serve as carbon precursors or functional groups [60]. Multifarious herbal medicines are rich in biological activities from polysaccharide [61], proteins [62], nucleic acids [63] and phospholipid [42], etc. These activities are the potent sources of functional groups (e.g., $\mathrm{C}, \mathrm{H}, \mathrm{N}$ and $\mathrm{O}$ ) without extra surface passivation or doping. For example, the hair-derived CDs are the only HMCDs that use human derivatives as a precursor [42]. The abundant microelements (e.g., phosphorus in phospholipid and sulfur in amino acid) tuned the intrinsic properties, enhancing the photoluminescence [42]. The nitrogen contents of hair CDs and skin CDs are higher than of citric acid CDs (Fig. 7). Besides, the protein is a biological macromolecule mainly includes chemical elements, such as carbon, hydrogen, oxygen and nitrogen. In light of the above discussions, these elements may contribute to the performance improvement of CDs. Therefore, the protein-rich animal drugs are expected to be an optimal precursor of HM-CDs synthesis.

\section{Synthetic methods of HM-CDs}

The methods of CDs synthesis fall into two categories: top-down and bottom-up strategies [64-67]. The former refers to large particles broken by physical, 


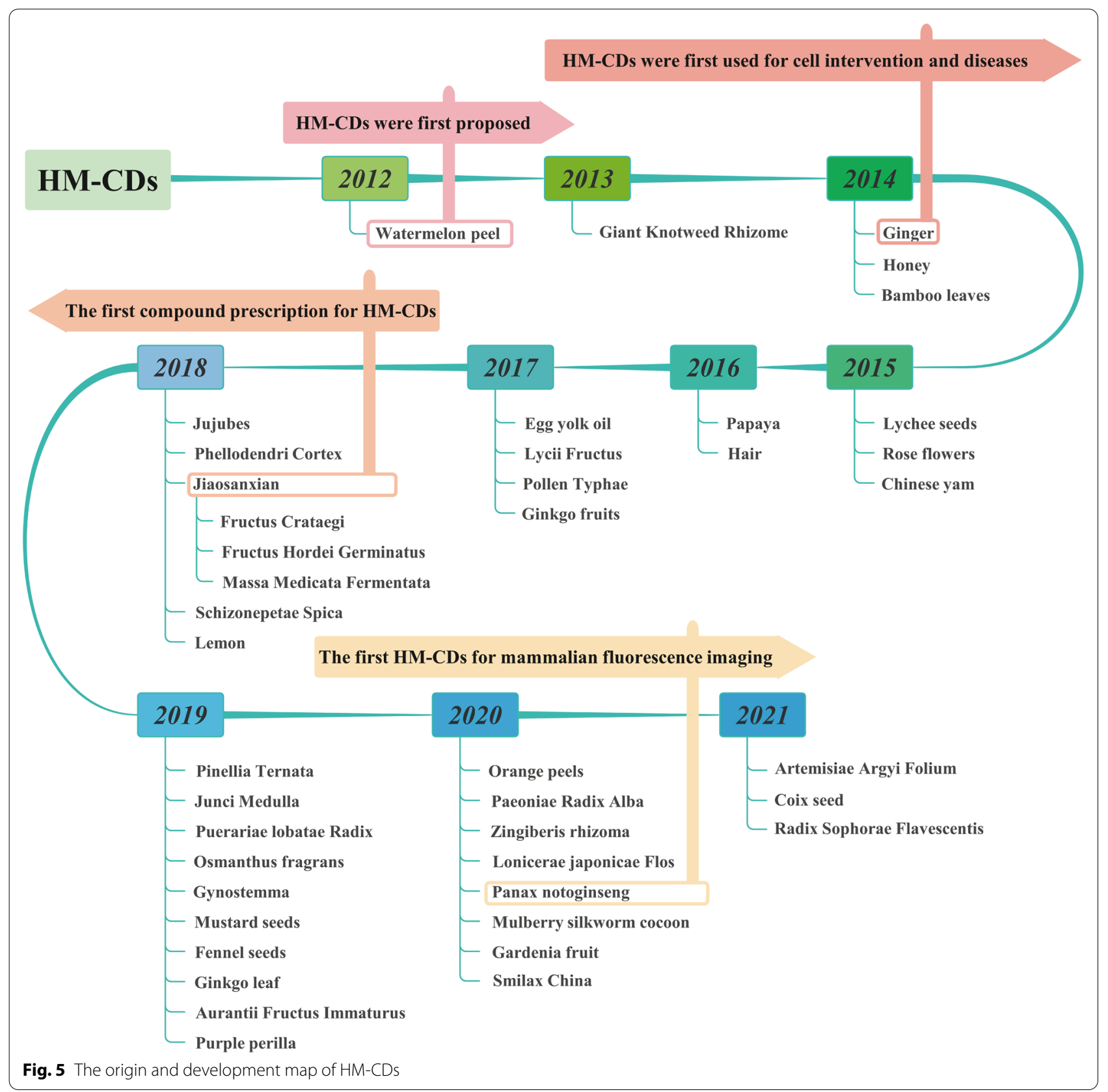

chemical or electrochemical technologies [23, 68], while the latter means nanoparticles formed by small molecules [23, 69, 70]. By contrast, bottom-up methods are more popular with scientists, because this technology shows double advantage of being straightforward and economical [71]. Bottom-up technique involves hydrothermal method [72], high-temperature pyrolysis method [73], solvothermal method [74] and microwavebased method [75]. Of these methods, hydrothermal synthesis is considered the greenest way to prepare HM-CDs [23, 76] (Table 2).

\section{Hydrothermal method \\ Synthesis procedure}

Hydrothermal synthesis is green without adding organic matter [77], that is the primary reason for preparing the CDs derived from natural substances. The surface of CDs does not require additional passivation to maximize safety and minimize toxicity. Before preparation, dried 


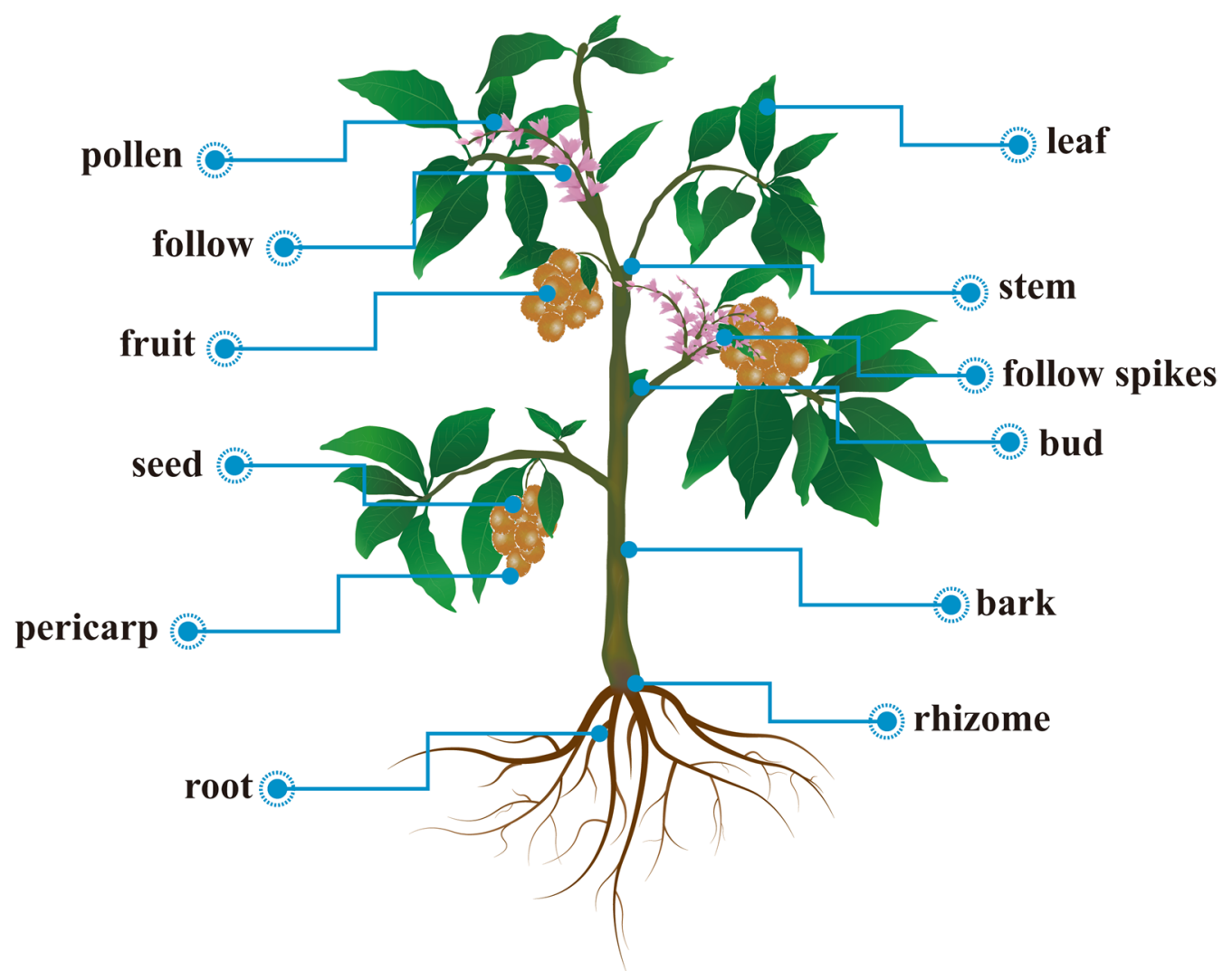

Fig. 6 Schematic diagram of medicinal parts of herbal medicine as precursors

herbs are cut into small pieces or powder in ultrapure water. After sonication, the mixture is transferred to a Teflon-lined stainless steel autoclave and heated at a specific temperature. To obtain pure CDs, the suspension needs further filtration with a $0.22 \mu \mathrm{m}$ cellulose filtration membrane and dialyzation with a dialysis bag for several days. (Fig. 8a).

\section{Reaction temperature and time}

Reaction temperature and time can affect the performance of HM-CDs. The hydrothermal reaction temperature of HM-CDs (such as CDs derived from papaya [78], yams [79], bamboo leaves [80]) was normally $100-200{ }^{\circ} \mathrm{C}$. $\mathrm{Li}$ et al. [81] synthesized the nitrogen-doped CDs from ginkgo fruits ( $\mathrm{H}-\mathrm{N}-\mathrm{CDs})$ at different temperatures and times. These CDs had the best fluorescence intensity, maximum quantum yield (QY) and lifetime (from the excited state to the ground state) when the temperature was set at $200{ }^{\circ} \mathrm{C}$. Another study [43] produced the honey-CDs at $100{ }^{\circ} \mathrm{C}$ for $2 \mathrm{~h}$. However, the CDs merely was stored steadily for 3 months at $4{ }^{\circ} \mathrm{C}$. The difference between the fluorescence intensity of honey-CDs was no longer significant when the synthesis time was extended to $12 \mathrm{~h}$ and even $16 \mathrm{~h}$, suggesting that the fluorescence intensity may have a saturated state. This phenomenon was also observed in coix seed CDs [82]. Specifically, the fluorescence intensity of coix seed-CDs decreased with the temperature rose from 15 to $80{ }^{\circ} \mathrm{C}$ [82]. The reason for this is that there exists the increased molecular collision frequency, non-radiative transition rate, as well as the constant radiation transition rate at high temperature [82]. Therefore, the CDs can function as a temperature sensor. The third representative research reported CDs derived from orange peel at different time points under the premise of the same temperature. This study noted that the QY decreased with the extension of reaction time, and the diameter increased slightly concurrently $[83,84]$.

\section{$H M-C D$ s derived from different medicinal parts and varieties}

CDs derived from the same part of different herbal species have distinct properties. The most typical study is that the scientists developed CDs derived from 14 different strains of orange peels. Different varieties gave rise to significant differences in QY under the same preparation 
Table 1 Herbal medicines for synthesizing HM-CDs

\begin{tabular}{|c|c|c|}
\hline Herbal medicine & Medical parts & Refs \\
\hline Pinellia ternata & Rhizome & [151] \\
\hline Osmanthus fragrans & Flower & {$[150,152]$} \\
\hline Mustard seeds & Seed & [157] \\
\hline Lemon juice & Fruit & {$[155]$} \\
\hline Ginkgo fruits & Seed & {$[81]$} \\
\hline Ginkgo leaf & Leaf & {$[85]$} \\
\hline Gynostemma & Whole herb & [161] \\
\hline Puerariae lobatae Radix & Root & [118] \\
\hline Schizonepetae Spica & Stem/flower spikes & {$[97,98]$} \\
\hline Papaya & Fruit & [78] \\
\hline Purple perilla & Leaf & {$[142]$} \\
\hline Ginger & Rhizome & {$[28]$} \\
\hline Aurantii fructus immaturus & Fruit & [117] \\
\hline Orange peel & Pericarp & {$[83,84,103]$} \\
\hline Lychee seeds & Seed & {$[96]$} \\
\hline Rose flowers & Flower & [153] \\
\hline Bamboo leaves & Leaf & {$[80]$} \\
\hline Phellodendri Chinensis Cortex & Bark & {$[110,127,162]$} \\
\hline Radix Sophorae Flavescentis & Root & {$[115]$} \\
\hline Lonicerae japonicae Flos & Flower & [116] \\
\hline Watermelon peel & Pericarp & {$[33]$} \\
\hline Gardenia fruit & Fruit & {$[88]$} \\
\hline Artemisiae Argyi Folium & Leaf & [134] \\
\hline Pollen Typhae & Pollen & [109] \\
\hline Panax notoginseng & Root & {$[38]$} \\
\hline Hair & Tissue & {$[42]$} \\
\hline Giant Knotweed Rhizome & Rhizome & [87] \\
\hline Junci Medulla & Whole herb & [163] \\
\hline Coix seed & Seed & {$[82]$} \\
\hline Smilax China & Root & [147] \\
\hline Yams & Rhizome & {$[79]$} \\
\hline Jujubes & Seed & [149] \\
\hline Honey & Honey & {$[43]$} \\
\hline Zingiberis rhizoma & Rhizome & [135] \\
\hline Fennel Seeds & Fruit & [94] \\
\hline Egg yolk oil & Egg yolk & [44] \\
\hline Lycii Fructus & Fruit & [148] \\
\hline Paeoniae Radix Alba & Root & [126] \\
\hline Jiaosanxian & Fruit/bud & [132] \\
\hline Mulberry silkworm cocoon & Whole worm & {$[45]$} \\
\hline
\end{tabular}

conditions [84]. This study further highlights that the QY may be associated with the amounts of volatile oils, which would drive the research of HM-CDs derived from the pericarp.

What's more, CDs extracted from different parts of the same herbal medicine also possess divergent performances. For instance, two HM-CDs derived from different parts of the same ginkgo tree were discovered $[81,85]$. The research team [85] prepared ginkgo leavesCDs with higher QY (22.80\%) using hydrothermal synthesis. In contrast, the QY of ginkgo fruits-CDs was only $3.33 \%$ in the same synthetic way [81]. The evidence demonstrates that herbal medicine from various parts of the same plant leads to considerable discrepancies of HMCDs, that may be due to the differential components.

\section{Size and blood-brain barrier permeability}

Biological barriers, such as the blood-brain barrier (BBB), hinder the infiltration of herbal macromolecules [86]. To overcome this problem, the world pays more attention to HM-CDs whose size is nanometer scale. In the hydrothermal synthesis, except for Giant Knotweed Rhizome-CDs [87], the average HM-CDs particle size was less than $10 \mathrm{~nm}$ (Table 2). The minimal average diameter was $2.08 \mathrm{~nm}$ [88]. The nanoscale HM-CDs prominently ameliorate the permeability, exerting more strengths than herbal medicine. The BBB penetration mechanism is classified into active transport and passive transport [89]. In the HM-CDs, one report noted that Pn-CDs could cross the BBB, which may be due to the ultra-small size, abundant surface functional groups, and the strong affinity to endothelial cell membrane of BBB [38]. But the molecular uptake mechanisms of Pn-CDs should be further evaluated. Furthermore, the CDs are propitious to the delivery of macromolecules via the carrier-mediated transport by covalently binding with drugs. Thus, these features enable the CDs improve the BBB permeability through passive transport [90]. Ashrafizadeh et al. [26] summarized the novel neuro-drug delivery systems for various neurological disorders using functionalized CDs as carriers. However, expensive ligands for modification limit their broad applications [26]. Herbal medicine is prescribed chronically for neurological disorders [91]. As a result, HM-CDs may enhance the BBB permeability of some macromolecules under non-functionalized conditions. It has the potential to be a novel tactic for herbal medicine to overcome biological barriers.

\section{High-temperature pyrolysis method Synthesis procedure}

High-temperature pyrolysis is a more common method in addition to hydrothermal synthesis. The organic substances in the precursors can be gradually converted into CDs via heating, dehydration, degradation and carbonization under high temperature in either vacuum or inert atmospheres throughout synthesis [92]. The process is facile, solvent-free, low-cost, and amenable to largescale production [93]. Herbal medicine is first put into the crucible and heated at a specific temperature with 

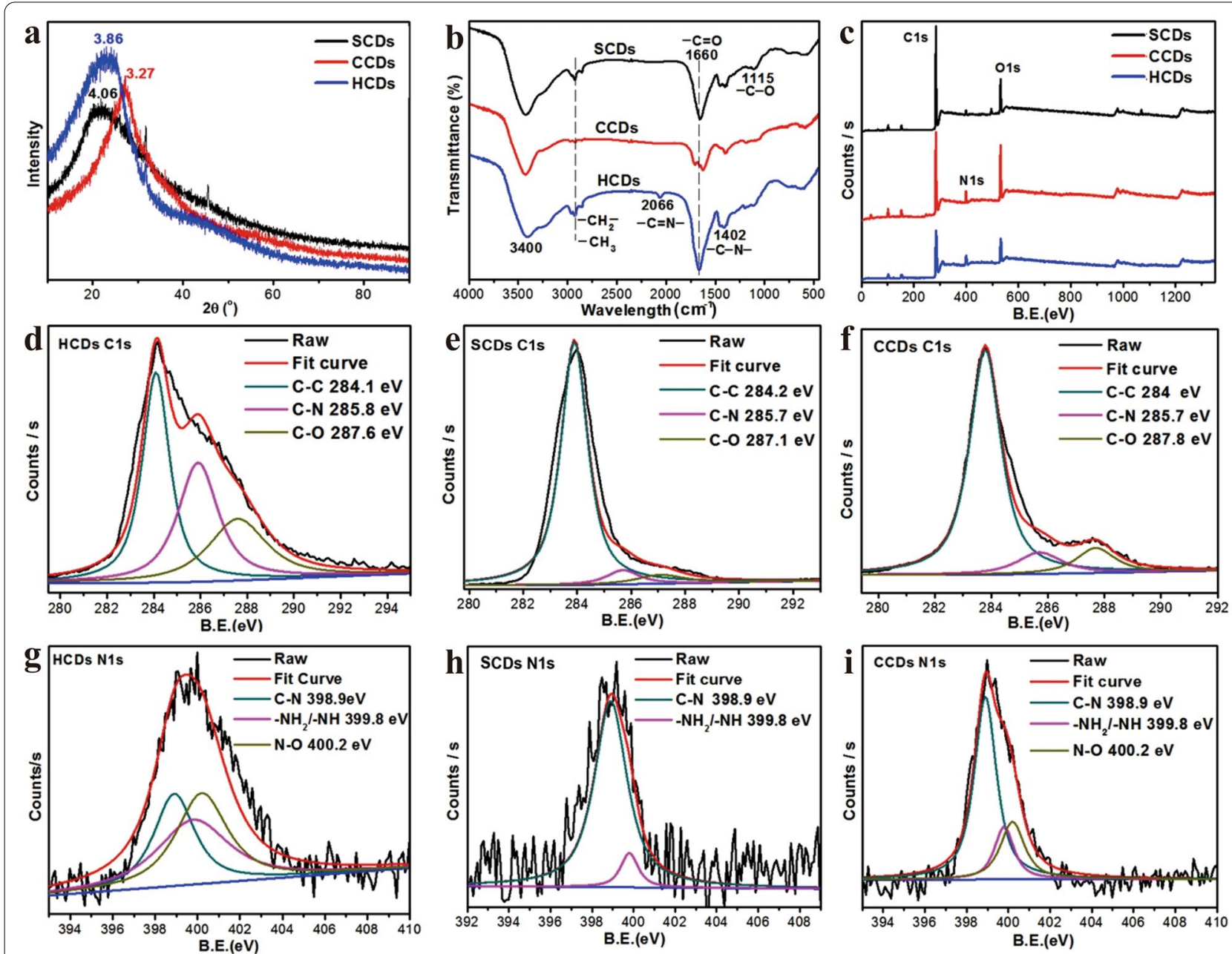

Fig. 7 Characterization of chemical structure of hair-CDs (HCDs), skin-CDs (SCDs) and citric acid-CDs (CCDs). a XRD patterns, b Fourier transform infrared spectra, c XPS spectra, $\mathbf{d}$ C1s profile of HCDs, e C1s profile of SCDs, $\mathbf{f}$ C1s profile of CCDs, $\mathbf{g}$ N1s profile of HCDs, $\mathbf{h}$ N1s profile of SCDs, i N1s profile of CCDs. Reprinted with permission from ref. [42]. Copyright (2016) Springer Nature

the muffle furnace until it was carbonized. The charred medicine is then crushed and boiled in ultrapure water, and the upper liquid is collected. After filtration through a $0.22 \mu \mathrm{m}$ microporous membrane, the solution is dialyzed using a dialysis bag for several days to harvast the purified CDs (Fig. 8b).

\section{Comparison of high-temperature pyrolysis and hydrothermal methods}

Compared with hydrothermal synthesis, high-temperature pyrolysis usually requires a higher reaction temperature (around $300{ }^{\circ} \mathrm{C}$ ). Concomitantly, the reaction temperature is higher, the heating time shorter (Table 2). Dager et al. [94] prepared a mono-dispersed CDs using the fennel seeds at a constant temperature of $500{ }^{\circ} \mathrm{C}$ for $3 \mathrm{~h}$. These CDs were stored for up to 15 months and had excellent colloidal solubility, photostability and environmental stability. In the existing HM-CDs prepared by the high-temperature pyrolysis, the minimum heating temperature was $220^{\circ} \mathrm{C}$. Blue-light CDs were prepared with watermelon peel as a carbon source at this temperature and dissolved in several solvents [33]. Another vital issue is the particle size of HM-CDs synthesized by both of the methods. While studies pointed out that the hydrothermal synthesis was easier to achieve the narrow size distribution of CDs than the pyrolysis [95], the latter can also achieve a smaller size. After checking statistical literature, we find that the diameters of HM-CDs prepared by pyrolysis are about $5 \mathrm{~nm}$ under the existing synthetic conditions (Fig. 9). It seemingly represents no noticeable difference in the particle diameters of HM-CDs synthesized by the two approaches. 
Table 2 Synthesis of HM-CDs

\begin{tabular}{|c|c|c|c|c|c|c|c|}
\hline Sources & Synthetic methods & $\begin{array}{l}\text { Reaction } \\
\text { temperature } \\
\left({ }^{\circ} \mathrm{C} / \mathrm{W}\right)\end{array}$ & Reaction time & QY (\%) & $\begin{array}{l}\text { Average } \\
\text { particle size } \\
(\mathrm{nm})\end{array}$ & $\begin{array}{l}\text { Particle size distribution } \\
(\mathrm{nm})\end{array}$ & Refs \\
\hline Pinellia Ternata & Hydrothermal & 180 & $9 \mathrm{~h}$ & 21.30 & 5.2 & $5.0-6.0$ & [151] \\
\hline Osmanthus fragrans & Hydrothermal & 180 & $10 \mathrm{~h}$ & 21.90 & 3.1 & $2.0-6.0$ & [152] \\
\hline Osmanthus fragrans & Hydrothermal & 240 & $5 \mathrm{~h}$ & 18.53 & 2.23 & $1.0-3.5$ & [150] \\
\hline Mustard seeds & Hydrothermal & 180 & $4 \mathrm{~h}$ & 4.60 & $4.58 \pm 0.26$ & $2.0-8.0$ & [157] \\
\hline Lemon juice & Hydrothermal & 120 & $3 \mathrm{~h}$ & 16.70 & 3.1 & $2.0-4.5$ & [155] \\
\hline Ginkgo fruits & Hydrothermal & $160 / 180 / 200$ & $8 / 12 / 16 \mathrm{~h}$ & 3.33 & 3.81 & $2.0-6.0$ & {$[81]$} \\
\hline Ginkgo leaf & Hydrothermal & 200 & $10 \mathrm{~h}$ & 22.80 & 3 & $2.0-4.0$ & {$[85]$} \\
\hline Papaya & Hydrothermal & 200 & $5 \mathrm{~h}$ & 18.98 & 3.4 & $2.0-6.0$ & {$[78]$} \\
\hline Purple perilla & Hydrothermal & 260 & $5 \mathrm{~h}$ & 9.01 & 2.8 & - & [142] \\
\hline Orange peel & Hydrothermal & 200 & $4 \mathrm{~h}$ & 18.57 & 2.34 & - & {$[84]$} \\
\hline Orange peel & Hydrothermal & 200 & $6 \mathrm{~h}$ & 11.37 & $2.9 \pm 0.5$ & - & [83] \\
\hline Bamboo leaves & Hydrothermal & 200 & $6 \mathrm{~h}$ & 7.10 & 3.6 & $3.4-4.2$ & {$[80]$} \\
\hline Panax notoginseng & Hydrothermal & 180 & $12 \mathrm{~h}$ & 8.54 & 7.8 & $7.2-8.4$ & {$[38]$} \\
\hline Panax notoginseng & Hydrothermal & 180 & $12 \mathrm{~h}$ & 18.41 & 6.66 & $5.58-7.75$ & {$[38]$} \\
\hline Giant Knotweed Rhizome & Hydrothermal & 200 & $3 \mathrm{~h}$ & 11.50 & 35 & - & {$[87]$} \\
\hline Jujubes & Hydrothermal & 190 & $6 \mathrm{~h}$ & - & 3.12 & - & [149] \\
\hline Honey & Hydrothermal & 100 & $2 \mathrm{~h}$ & - & - & Mainly $2 \mathrm{~nm}$ & {$[43]$} \\
\hline Lycii Fructus & Hydrothermal & 180 & $5 \mathrm{~h}$ & 17.20 & 3.3 & $2.0-5.0$ & [148] \\
\hline Ginger & Hydrothermal & 300 & $2 \mathrm{~h}$ & 13.40 & $4.3 \pm 0.8$ & - & {$[28]$} \\
\hline Yams & Hydrothermal & 200 & $2 \mathrm{~h}$ & - & - & $1.5-4.0$ & [79] \\
\hline Gardenia fruit & Hydrothermal & 180 & $5 \mathrm{~h}$ & 10.70 & 2.08 & $1.2-3.5$ & {$[88]$} \\
\hline Coix seed & Hydrothermal & 180 & $3 \mathrm{~h}$ & 17.43 & $2.4 \pm 0.2$ & $2.1-2.7$ & {$[82]$} \\
\hline Smilax China & Hydrothermal & 200 & $5 \mathrm{~h}$ & 22.37 & $2.10 \pm 0.29$ & $0.5-3.0$ & [147] \\
\hline Puerariae lobatae Radix & $\begin{array}{l}\text { High-temperature } \\
\text { pyrolysis }\end{array}$ & 300 & $1 \mathrm{~h}$ & 3.20 & - & $3.0-10.0$ & [118] \\
\hline Schizonepetae Spica & $\begin{array}{l}\text { High-temperature } \\
\text { pyrolysis }\end{array}$ & 350 & $1 \mathrm{~h}$ & 6.31 & - & $1.29-6.87$ & [98] \\
\hline Schizonepetae Spica & $\begin{array}{l}\text { High-temperature } \\
\text { pyrolysis }\end{array}$ & 350 & $1 \mathrm{~h}$ & 2.26 & - & $0.8-4.0$ & [97] \\
\hline Aurantii fructus immaturus & $\begin{array}{l}\text { High-temperature } \\
\text { pyrolysis }\end{array}$ & 350 & $1 \mathrm{~h}$ & 7.20 & - & $1.1-4.4$ & [117] \\
\hline Lychee seeds & $\begin{array}{l}\text { High-temperature } \\
\text { pyrolysis }\end{array}$ & 300 & $2 \mathrm{~h}$ & 10.60 & 1.12 & $0.4-2.0$ & [96] \\
\hline $\begin{array}{l}\text { Phellodendri Chinensis } \\
\text { Cortex }\end{array}$ & $\begin{array}{l}\text { High-temperature } \\
\text { pyrolysis }\end{array}$ & 350 & $1 \mathrm{~h}$ & - & $2.84 \pm 0.89$ & - & [127] \\
\hline $\begin{array}{l}\text { Phellodendri Chinensis } \\
\text { Cortex }\end{array}$ & $\begin{array}{l}\text { High-temperature } \\
\text { pyrolysis }\end{array}$ & 350 & $1 \mathrm{~h}$ & 9.62 & - & $1.2-4.8$ & [110] \\
\hline $\begin{array}{l}\text { Phellodendri Chinensis } \\
\text { Cortex }\end{array}$ & $\begin{array}{l}\text { High-temperature } \\
\text { pyrolysis }\end{array}$ & 400 & $1 \mathrm{~h}$ & 5.63 & $1.93 \pm 0.53$ & $0.5-3.6$ & [162] \\
\hline Radix Sophorae Flavescentis & $\begin{array}{l}\text { High-temperature } \\
\text { pyrolysis }\end{array}$ & 350 & $1 \mathrm{~h}$ & 1.08 & - & Mainly 2.0-3.0 & [115] \\
\hline Lonicerae japonicae Flos & $\begin{array}{l}\text { High-temperature } \\
\text { pyrolysis }\end{array}$ & 350 & $1 \mathrm{~h}$ & 0.50 & - & $1.0-10.0$ & [116] \\
\hline Watermelon peel & $\begin{array}{l}\text { High-temperature } \\
\text { pyrolysis }\end{array}$ & 220 & $2 \mathrm{~h}$ & 7.10 & $2.0 \pm 0.5$ & - & [33] \\
\hline Artemisiae Argyi Folium & $\begin{array}{l}\text { High-temperature } \\
\text { pyrolysis }\end{array}$ & 350 & $1 \mathrm{~h}$ & 0.19 & - & $6.0-10.0$ & [134] \\
\hline Pollen Typhae & $\begin{array}{l}\text { High-temperature } \\
\text { pyrolysis }\end{array}$ & 350 & $1 \mathrm{~h}$ & - & $4.2 \pm 1.4$ & $2.0-8.0$ & [109] \\
\hline Junci Medulla & $\begin{array}{l}\text { High-temperature } \\
\text { pyrolysis }\end{array}$ & 350 & $1 \mathrm{~h}$ & 0.12 & - & $1.0-8.0$ & [163] \\
\hline
\end{tabular}


Table 2 (continued)

\begin{tabular}{|c|c|c|c|c|c|c|c|}
\hline Sources & Synthetic methods & $\begin{array}{l}\text { Reaction } \\
\text { temperature } \\
\left({ }^{\circ} \mathrm{C} / \mathrm{W}\right)\end{array}$ & Reaction time & QY (\%) & $\begin{array}{l}\text { Average } \\
\text { particle size } \\
(\mathrm{nm})\end{array}$ & $\begin{array}{l}\text { Particle size distribution } \\
(\mathrm{nm})\end{array}$ & Refs \\
\hline Zingiberis rhizoma & $\begin{array}{l}\text { High-temperature } \\
\text { pyrolysis }\end{array}$ & 350 & $1 \mathrm{~h}$ & 5.20 & $3.00 \pm 0.77$ & $2.23-3.77$ & [135] \\
\hline Fennel Seeds & $\begin{array}{l}\text { High-temperature } \\
\text { pyrolysis }\end{array}$ & 500 & $3 \mathrm{~h}$ & 9.50 & $3.90 \pm 0.91$ & - & [94] \\
\hline Egg yolk oil & $\begin{array}{l}\text { High-temperature } \\
\text { pyrolysis }\end{array}$ & 260 & $1 \mathrm{~h}$ & 5.01 & - & Mainly less than $10 \mathrm{~nm}$ & [44] \\
\hline Paeoniae Radix Alba & $\begin{array}{l}\text { High-temperature } \\
\text { pyrolysis }\end{array}$ & 350 & $1 \mathrm{~h}$ & 5.34 & - & $1.0-2.4$ & [126] \\
\hline Jiaosanxian & $\begin{array}{l}\text { High-temperature } \\
\text { pyrolysis }\end{array}$ & - & - & 7.95 & - & $4.4-6.4$ & [132] \\
\hline Mulberry silkworm cocoon & $\begin{array}{l}\text { High-temperature } \\
\text { pyrolysis }\end{array}$ & 350 & $1 \mathrm{~h}$ & 6.32 & - & $2.26-9.35$ & [45] \\
\hline Gynostemma & $\begin{array}{l}\text { High-temperature } \\
\text { pyrolysis }\end{array}$ & 400 & $4 \mathrm{~h}$ & 5.7 & $2.49 \pm 0.43$ & - & [161] \\
\hline Hair & $\begin{array}{l}\text { High-temperature pyroly- } \\
\text { sis/microwave }\end{array}$ & $300 / 400$ & $2 \mathrm{~h} / 4 \mathrm{~min}$ & 86.06 & 3.57 & $2.0-6.0$ & {$[42]$} \\
\hline Rose flowers & Microwave & - & - & - & - & $4.0-6.0$ & [153] \\
\hline Ginkgo fruits & Microwave & 800 & $5 / 10 / 15 \min$ & 0.65 & 2.82 & $2.0-4.0$ & [81] \\
\hline Orange peel & Microwave & 900 & $1 \mathrm{~min}$ & 16.20 & 4.2 & $3.0-5.0$ & [103] \\
\hline Papaya & Solvothermal & 200 & $5 \mathrm{~h}$ & 18.39 & 10.8 & $8.0-18.0$ & [78] \\
\hline
\end{tabular}

\section{Quantum yield and particle size}

Unlike the previous review [93], QY of pyrolysis synthesis is lower than that of hydrothermal because of the diversity of carbon sources. Except for CDs with a high QY (10.60\%) synthesized from lychee seeds [96], the average QY of others was less than $10 \%$ (Table 2). Remarkably, as for the synthesis of Schizonepetae Herba CarbonisataCDs (SHC-CDs), two prior studies yielded divergent results under a uniform condition. Both of Zhang et al. [97] and Sun et al. [98] created SHC-CDs. The former reported a SHC-CDs with an average size of $0.8-4.0 \mathrm{~nm}$ and a QY of $2.26 \%$. While in the latter publication, the average size and QY of SHC-CDs were 1.29-6.87 nm and $6.31 \%$, respectively. These findings illustrate the destabilization of this approach. Optimal synthesis continues to be explored. By combining pyrolysis with microwave methods, Zhang and others [42] produced hair CDs with high QY (86.06\%), much higher than that of citric acid CDs (19.73\%). Except for differences in carbon sources, the combination of both synthetic strategies may offer potential benefits. They also fabricated skin CDs with higher QY (51.35\%), demonstrating that protein-rich materials are more suitable as precursors [42]. Hence, animal-derived herbal medicine may be the most promising drugs for HM-CDs synthesis with high QY in the future.

\section{Microwave method \\ Synthesis procedure}

The synthesis process of microwave is similar to that of pyrolysis. Differently, the former can reach the intended energy in a short time and ensure that the precursors are heated evenly. Moreover, it's an electromagnetic wave (wavelength range between $1 \mathrm{~mm}$ and $1 \mathrm{~m}$ ), which arises the cleavage of chemical bonds via delivering energy [99]. Thus, it enables us to fabricate uniform CDs by breaking chemical bonds. This method significantly reduces the response time and enhances the effectiveness of preparation [75, 100, 101]. (Table 2) Additionally, microwaveassisted hydrothermal synthesis has been reported as an alternative to traditional hydrothermal synthesis [102].

\section{Comparison with other methods}

To compare with other methods, Li et al. [81] prepared two ginkgo fruits-CDs (H-CDs/M-CDs) using hydrothermal $(\mathrm{H})$ and microwave $(\mathrm{M})$ methods, respectively. The synthesis of M-CDs took only 5-15 min, much shorter than that of hydrothermal method. The particle size was relatively smaller (Table 2 ). However, the fluorescence property of $\mathrm{H}$-CDs was much better than that of M-CDs. On one hand, it is because of H-CDs with more regular and uniform morphology. On the other hand, the luminescence mechanism plays an important 


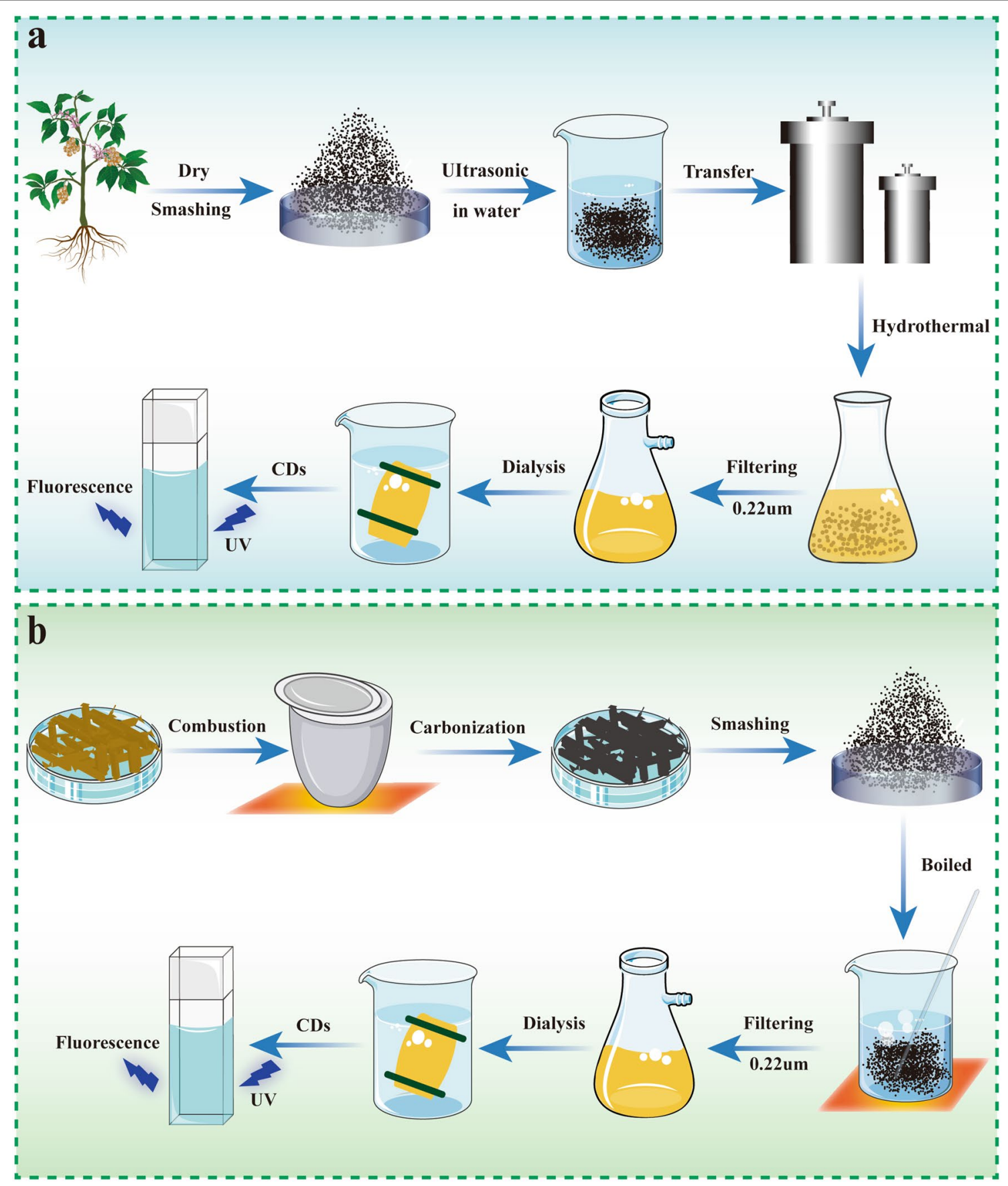

Fig. 8 Schematic diagram on the synthesis process of HM-CDs. a The hydrothermal synthesis process of HM-CDs, $\mathbf{b}$ High-temperature pyrolysis process of HM-CDs

role. The QY and lifetime of $\mathrm{H}$-CDs were greater than $\mathrm{M}-\mathrm{CDs}$ and had a more vigorous fluorescence intensity (Table 2). Notably, the microwave method could even prepare CDs derived from orange peel within $1 \mathrm{~min}$ and yield up to $16.20 \%$ [103]. These CDs had high green fluorescence with excitation-dependent emission fluorescence behavior. Indubitably, microwave synthesis may be better than the hydrothermal and pyrolysis methods according to reflection time and efficiency. Although the microwave method has the above positives, it remains the rare application for HM-CDs synthesis. 

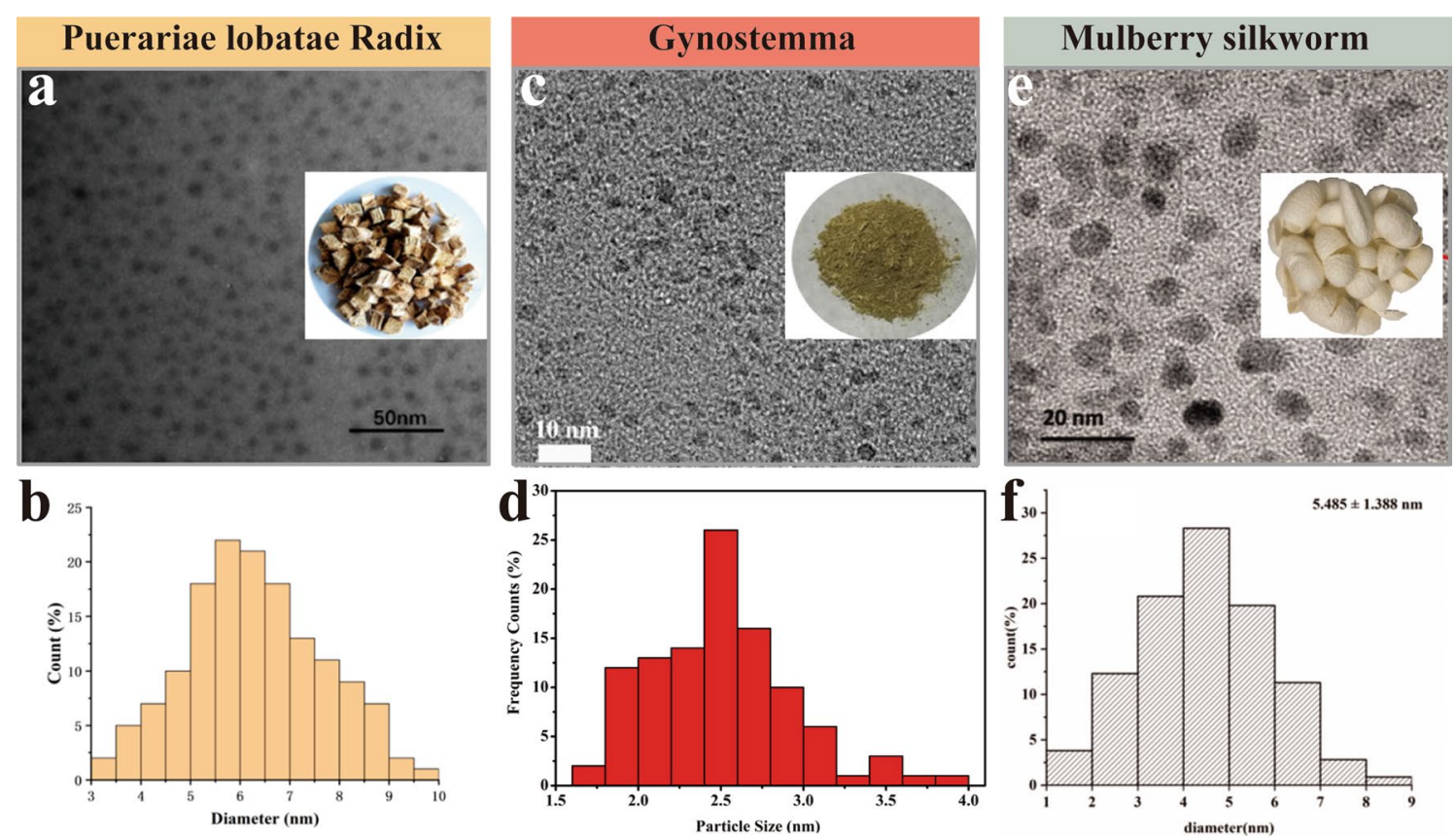

Fig. 9 Representative transmission electron microscopy (TEM) images of HM-CDs prepared by high-temperature pyrolysis. a HM-CDs are derived from Puerariae lobatae Radix (inset), b TEM size distribution of Puerariae lobatae Radix-CDs, c HM-CDs are derived from Gynostemma (inset), $\mathbf{d}$ TEM size distribution of Gynostemma-CDs, e HM-CDs are derived from the mulberry silkworm cocoon (inset), f $\mathrm{f}$ TEM size distribution of mulberry silkworm-CDs. Reprinted with permission from ref. [45, 118, 161]. Copyright (2019) by the authors. Licensee MDPI, Basel, Switzerland. Copyright (2019) American Chemical Society. Copyright (2019) The Authors. Published by Informa UK Limited

\section{Solvothermal method}

Unlike hydrothermal synthesis, the solvothermal method involves a variety of solvents other than water [54, 104]. Wang et al. [78] prepared ethanol-papaya CDs (E-CDs) and water-papaya CDs (W-CDs), respectively. There were a vast number of saccharides and a small number of water-soluble macromolecules in the water-based medium, all of which were beneficial to the production of W-CDs. Conversely, more organic macromolecules in $90 \%$ ethanol led to E-CDs with larger size [78]. As a result, the W-CDs had better fluorescence stability, exposing the ethanol defects.

Two most commonly methods are hydrothermal and high-temperature pyrolysis. While hydrothermal synthesis is called the greenest manner, the reaction time is relatively long. This drawback causes inefficiency of synthesis. Other methods are rarely selected to prepare HM-CDs currently, but have been pervasively used to synthesize CDs derived from other precursors, especially microwave. Short reaction time, high efficiency, heat uniformity and other characteristics will make the microwave method ideal for HM-CDs synthesis.

Collectively, the particle size of HM-CDs obtained by all methods were unevenly distributed. QY was generally low. The defects essentially hinder the utility in biomedicine and the realization of future commercialization.

\section{Applications of HM-CDs \\ Medical applications}

Nanomedicine has become a highly active research field [105-107]. The emergence of CDs contributes new strength to the development of nanomedical science. Currently, avoiding complicated modifications and expensive materials receives key concerns. Existing CDs can be only treated diseases either by loading pharmacophores or as drug carriers. Excitedly, herbal medicine precursors may overcome these limitations through their specific efficacy, which naturally catches the eye of investigators. In this part, we discuss the diseases treated by existing HM-CDs and the specific functional mechanisms. (Fig. 10).

\section{Hemostasis}

Charcoal drugs, an essential component of herbal medicine, have more than 2000 years in China [108]. Although the hemostasis effects of charcoal drugs are broadly recognized, the material basis from the perspective of small molecule activators is poorly understood [108, 109]. The carbonization is completed by heating at high 

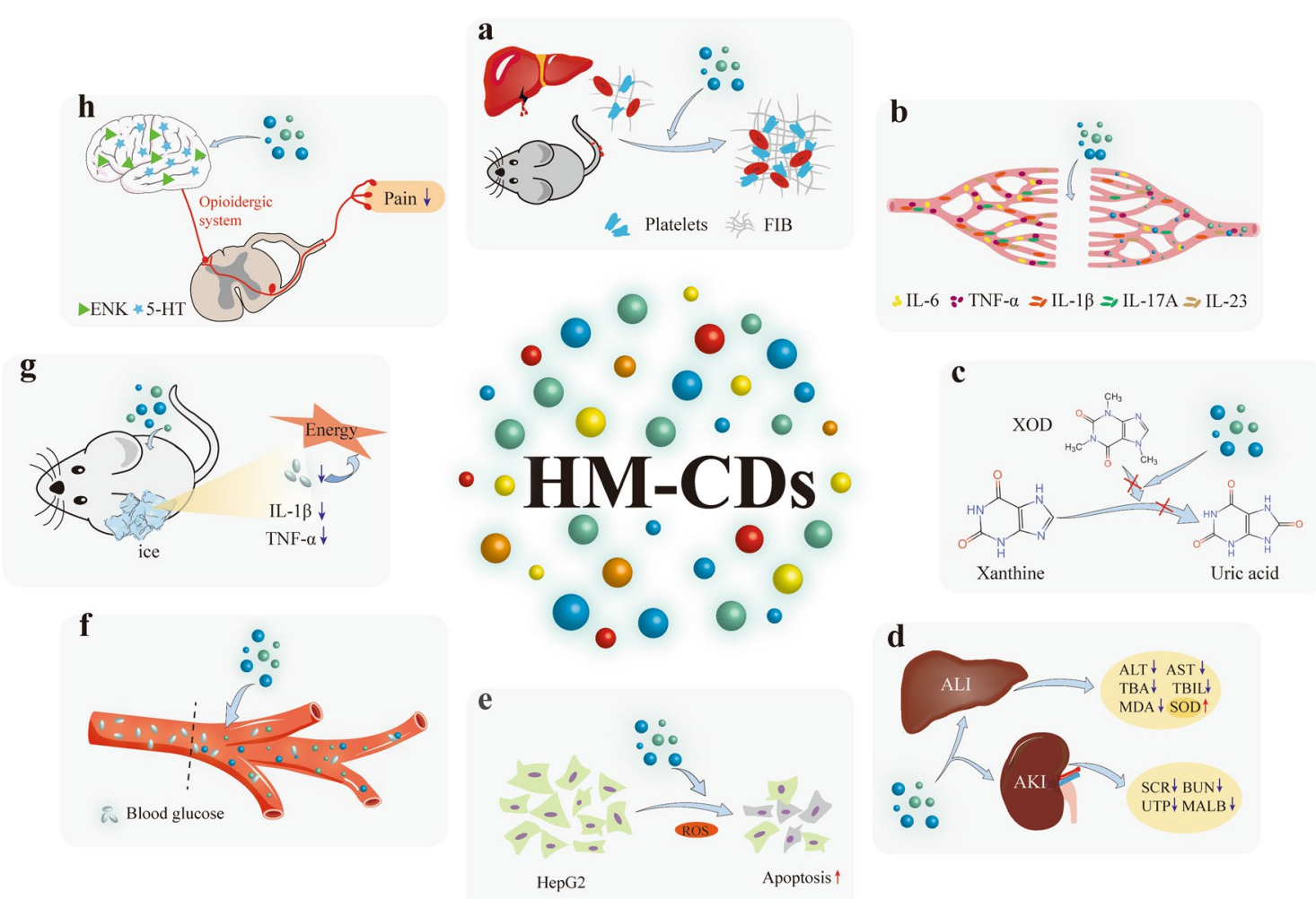

Fig. 10 Medical applications and potential mechanisms of HM-CDs. a Hemostasis of HM-CDs, b Anti-inflammatory of HM-CDs, c Anti-hyperuricemia of HM-CDs. d Hepato-renal protective effect of HM-CDs, e Anticancer of HM-CDs, $\mathbf{f}$ Hypoglycemic of HM-CDs, $\mathbf{g}$ Anti-frostbite of HM-CDs, $\mathbf{h}$ Analgesic of HM-CDs. FIB: fibrinogen; XOD: xanthine oxidase; ALT: alanine transaminase; AST: acetone transaminase; TBA: total bile acid; TBIL: total bilium; MDA: malondialdehyde; SOD: superoxide dismutase; SCR: serum creatinine; BUN: blood urea nitrogen; UTP: urine total protein; MALB: microalbuminuria; ER: endoplasmic reticulum; ROS: reactive oxygen species; ENK: enkephalin; 5-HT: serotonin

temperature, which is one of the operations of pyrolysis and microwave methods. HM-CDs can be prepared after the charcoal herbs are boiled, filtered and dialysis. In view of this, researchers turned their attention to the novel products, and simultaneously elucidated the specific mechanisms of HM-CDs in hemostasis.

Junci Medulla Carbonisat, Pollen Typhae Carbonisata and Schizonepetae Herba Carbonisata have long been incorporated into the hemorrhagic carbonized herbal medicine in the treatment of hemorrhagic diseases for hundreds of years. The experiments on tail haemorrhage and liver haemorrhage revealed that SHC-CDs and Pollen Typhae Carbonisata-CDs (PTC-CDs) attenuated activated partial thromboplastin time (APTT) and increased fibrinogen (FIB), indicating that they can exert hemostatic effects by activating the internal coagulation system and FIB $[97,109]$. Another study showed that SHC-CDs may inhibit bleeding by increasing platelets [98]. In addition to activation of FIB system, the Junci Medulla Carbonisata-CDs (JMC-CDs) activate the exogenous coagulation system. It provides a perspective to explore the mechanisms of charred herbal medicine with hemostatic effects.

Apart from charcoal drugs, herbal medicine that dose not perform hemostatic effects can be prepared into CDs by pyrolysis, endowing them with additional hemostatic function. For example, egg yolk oil-CDs (EYO-CDs) suppressed bleeding by activating the intrinsic coagulation pathways and FIB system in a dose-dependent manner [44]. Another work [110] demonstrated that low doses of Phellodendri Cortex Carbonisatus-carbon dots (PCCCDs) $(1 \mathrm{mg} / \mathrm{kg})$ significantly cut down thrombin time, and had an excellent hemostatic effect, thus reduced PCC usage in vivo.

\section{Anti-inflammatory}

Inflammation, a complex pathological process of multipathways and multi-molecules, corresponds to the pharmacological effects of multi-components and multitargeting herbal medicines [111, 112]. Herbal medicine is known as a promising therapy to carry out anti-inflammation [113]. Despite increased anti-inflammatory bioactive substances from herbal medicine [114], the 


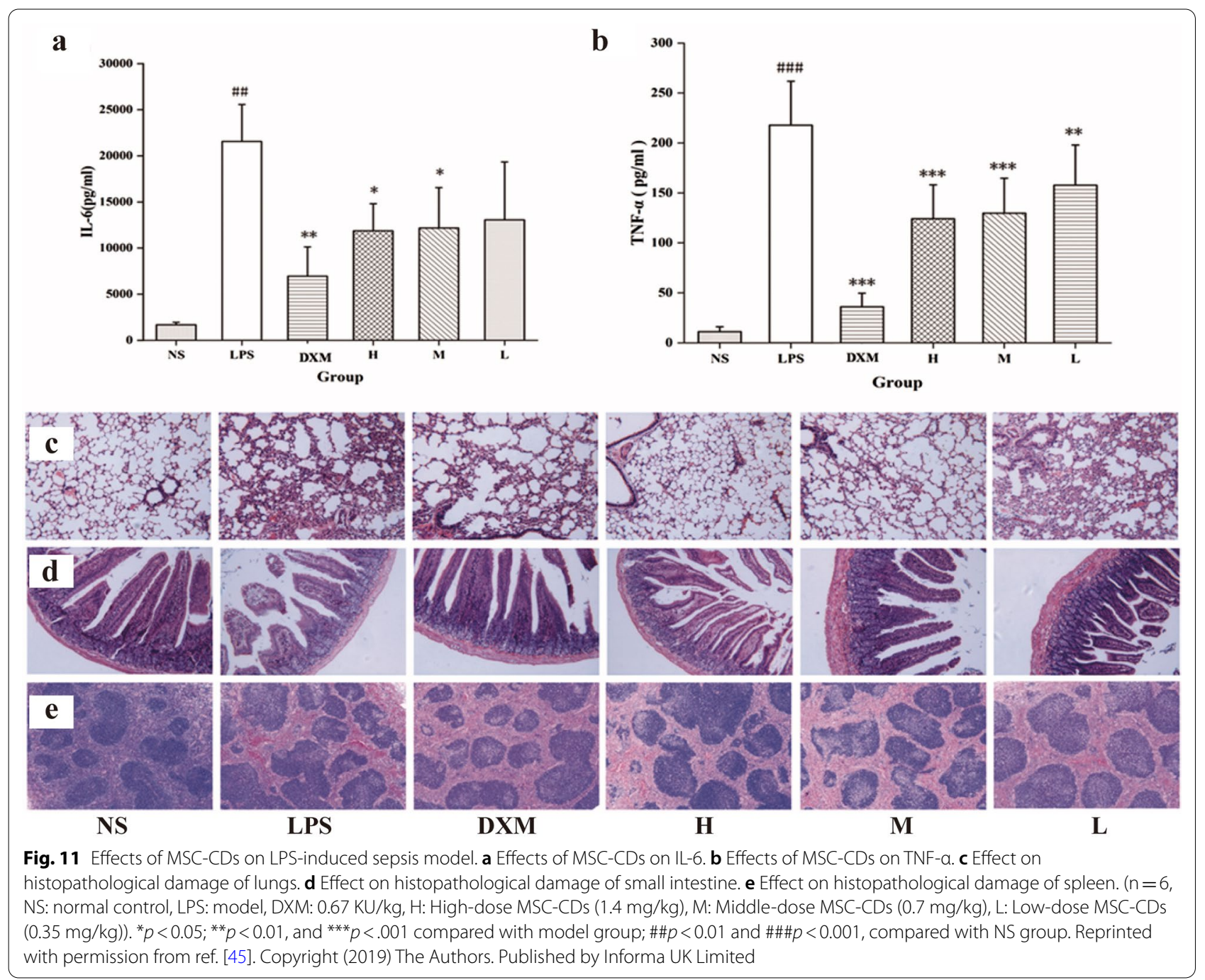

underlying mechanisms still needs to be lucubrated. To date, mulberry silkworm cocoon-CDs (MSC-CDs), Lonicerae japonicae Flos-derived CDs (LJFC-CDs), Aurantii fructus immaturus carbonisata-derived CDs (AFIC-CDs) and Puerariae lobatae Radix CDs (PLR-CDs) were produced through pyrolysis. Among these HM-CDs, MSCCDs displayed favorable anti-inflammatory effects in the xylene-induced ear edema and acetic acid-induced vascular permeable mouse models, which provides clear evidence for inflammatory treatment related to vascular endothelial barrier leakage and cytokine release. More attractively, in the LPS-induced systemic inflammation model, MSC-CDs attenuated serum levels of IL- 6 and TNF- $\alpha$ by dose-dependence [45] (Fig. 11).

Recently, Hu et al. [115] showed Radix Sophorae Flavescentis carbonisata (RSFC)-based CDs against an ethanol-induced acute gastric ulcer in rats by inhibiting
TNF- $\alpha$ and IL- 6 releases via downregulation of NF- $\kappa B$ pathway. RSFC has been extensively harvested for treating ulcerative diseases throughout the body. The authors concluded that HM-CDs using high-temperature pyrolysis may have inherent bioactivities [115]. But the active ingredients are not revealed.

With regard to anti-inflammation induced by LJFCCDs, they markedly declined levels of IL-1 $\beta$, IL- 6 and TNF- $\alpha$ in LPS-induced heating models [116]. Besides, AFIC-CDs [117] and PLR-CDs [118] ameliorated the degree of joint swelling in gouty arthritis, and the former reduced the levels of IL-1 $\beta$ and TNF- $\alpha$ in a dose-dependent manner. Furthermore, treatment of psoriasis-like inflammation with PCC-CDs not only reduced the IL- 6 and TNF- $\alpha$, but also decreased the IL-17A and IL-23 levels. These findings offer a novel approach for anti-inflammatory of herbal medicine. 
a

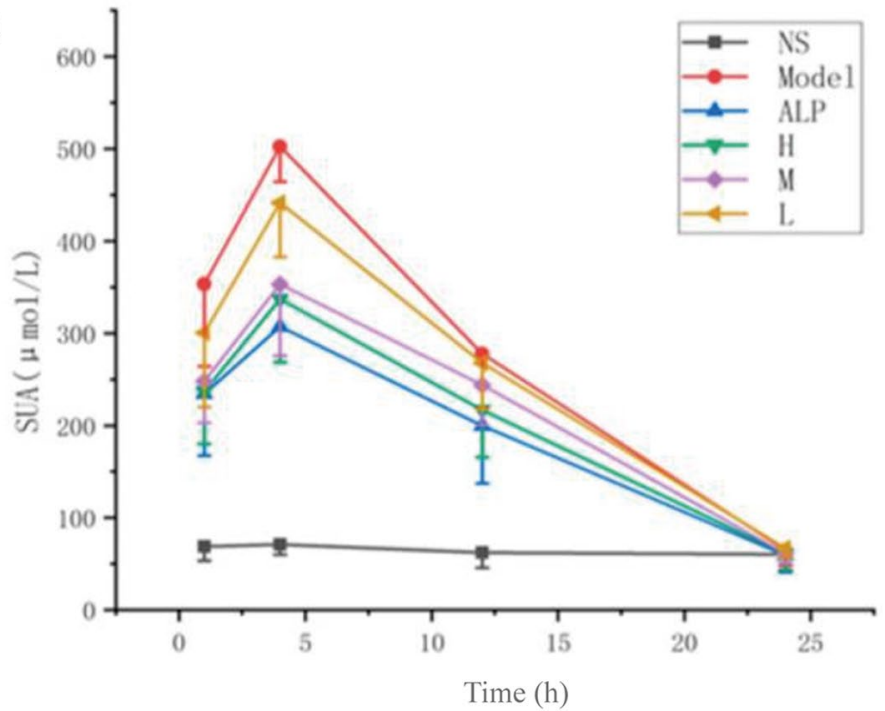

C
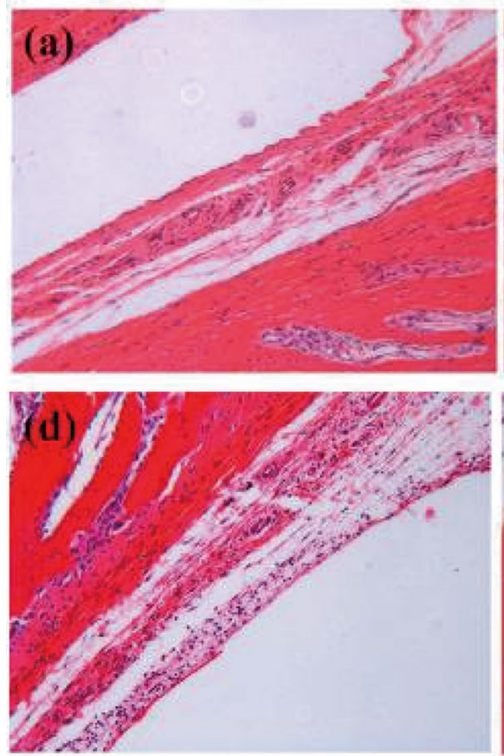
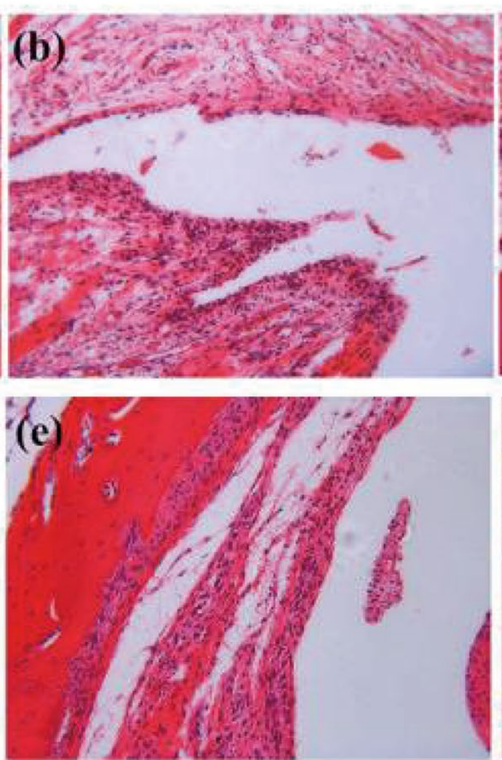

b

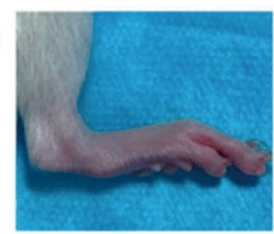

NS

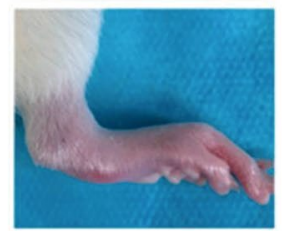

Model

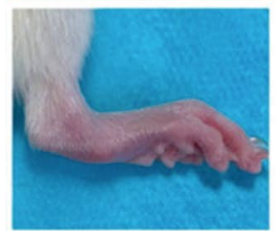

$\mathrm{H}$
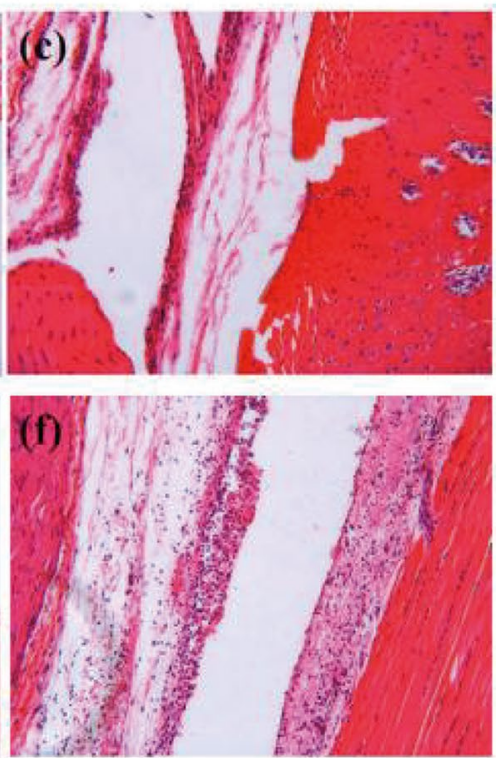

Fig. 12 Anti-Gout effects of PLR-CDs. a Effects of PLR-CDs on serum uric acid levels for $24 \mathrm{~h}$. $\mathbf{b}$ Representative images of joints from NS, Model, and $\mathrm{H}$ groups at $12 \mathrm{~h}$. $\mathbf{c}$ Histological examination of H \& E stained ankle joint tissues of normal group (a), model group (b), Col group (c), High-dose PLR-CDs group (d), Middle-dose PLR-CDs group (e), and Low-dose PLR-CDs group (f). Reprinted with permission from ref. [118]. Copyright (2019) by the authors. Licensee MDPI, Basel, Switzerland

\section{Anti-hyperuricemia}

Hyperuricemia is one of the pathological processes of gyration mainly associated with purine metabolic disorder and higher serum urate [119, 120]. The elevation of xanthine oxidase (XOD) is responsible for the pathological basis for the overproduction of uric acid in the kidneys. Allopurinol, an inhibitor of XOD, currently becomes a first-line drug for clinical treatment of gout and hyperuricemia [121]. Nevertheless, skin rash is the most common adverse effect of allopurinol, increasing high mortality $[122,123]$. For the treatments, AFIC-CDs and PLR-CDs prepared by Zhao et al. $[117,118]$ reduced uric acid levels in a short period (Fig. 12), and also decreased inflammation during the acute phase of gout. Particularly, the non-toxicity of CDs effectively avoided the potentially toxic side effects of allopurinol [124]. Therefore, it offers a safer and more reliable regimen for gout and hyperuricemia in clinical practice. 


\section{Hepato-renal protective effect}

In western countries, herbal medicine is often considered adequate only for chronic diseases and slow or ineffective for acute injuries [125]. However, HM-CDs confirmed the therapeutic effects of herbal medicine in acute injuries. In a latest study [126], the Paeoniae Radix Alba Carbonisata-derived CDs (PRAC-CDs) diminished alanine transaminase (ALT) and acetone transaminase (AST), and inhibited total bile acid (TBA) and total bilium (TBIL) in mice model of acute liver injury. The CDs led to a hepatoprotective effects via a decrease in Malondialdehyde (MDA) content and an increase in superoxide dismutase (SOD) by removing oxygen freelance, inhibiting lipid peroxidation of liver cells, and regulating the metabolism of bile acid. Another study revealed that PCC-CDs directly exerted renal-protection reversing increases of serum creatinine (SCR), blood urea nitrogen (BUN), total urine protein (UTP) and microalbuminuria (MALB) in acute kidney injury models [127]. The PCC-CDs also mitigated the inflammatory responses and thrombocytopenia associated with acute kidney injury and thereby acted multifacetedly.

\section{Anticancer}

Herbal medicine has been diffusely used in the alternative treatment of cancer, and further plays an auxiliary therapeutic role by regulating cancer genes and anti-cancer genes, epigenetic modifications, and tumor microenvironment [128-130]. HM-CDs execute anti-cancer effects which may be a new form for positioning tumors by photoluminescence. Following hydrothermal synthesis, Ginger-CDs excited strong blue, green and red fluorescence at different wavelengths [28]. Compared with CDs synthesized by EDTA, glycine and green tea, ginger-CDs selectively induced apoptosis by inducing ROS production in HepG2 cells [28]. This pharmacologic action was dependent on the surface modification of ginger-CDs. Simultaneously, ginger-CDs were excreted by urine within $1 \mathrm{~h}$, and not be stored in large quantities in the body, resulting in avoidance of potential biotoxicity. Unexpectedly, the surface composition of ginger-CDs did not contain the 6-gingerol of the anti-cancer active component except curcumin [28]. On basis of this, some substances may be changed after high-temperature heating can be inferred, leading to diverse pharmacological effects between HM-CDs and herbal medicines. Hence, researchers need to continually explore and validate active pharmaceutical ingredients of HM-CDs. It must be pointed that few publications are reported on tumor bioimaging applications of HM-CDs. Part of the reason may lie in the deficiency of red HM-CDs required for bioimaging.

\section{Hypoglycemic}

Jiaosanxian, a charcoal herbal medicine, has the effect of eliminating food mass. The postprandial glycemia and insulin levels are inversely proportional to carbohydrate degradation rate [131]. So JSX-CDs were synthesized to lower blood glucose levels [132]. Fifteen minutes after gavage, JSX-CDs markedly reduced the blood glucose level of hyperglycemic mice. Although there was no significant difference between the JSX-CDs and control groups after $90 \mathrm{~min}$, blood glucose level was still lower than those in the control group. Preliminary results revealed that JSX-CDs did not cause hypoglycemia in normal mice. But the underlying mechanism of action is still not precise.

\section{Anti-frostbite}

Frostbite caused by cold conditions triggers various degrees of damage to tissues, but interventions are lacking [133]. To bridge this gap, Kong et al. [134] synthesized Artemisiae Argyi Folium (AAF) Carbonisata-CDs (AAFC-CDs) by pyrolysis method. AAFC-CDs achieved anti-frostbite efficacy by mediating IL- $1 \beta$ and TNF- $\alpha$ to improve local inflammation and providing energy for the body to reduce blood glucose levels caused by frostbite (Fig. 13). Unlike traditional AAF, isochlorogenic acid no longer existed in AAFC-CDs, but the specific components have not been identified. Traditional AAF was not indicated for treating frostbite previously. Hence, the appearance of AAFC-CDs may expand the practical applications of AAF.

\section{Analgesic}

The analgesic activity of Zingiberis Rhizoma (Ganjiang, ZR), a processed product of ginger, has been for millennia. Zhang et al. [135] prepared the ZR-CDs by the high-temperature pyrolysis. ZR-CDs were comparable to the analgesic activity of morpholino [136]. The analgesic mechanisms on the following two aspects. First, ZR-CDs could enhance endogenous opioid peptide enkephalin (ENK), which was abrogated with non-selective antagonist naloxone, indicating that the activation of opioid system was one of the analgesic effects. Second, ZRCDs increased serotonin (5-HT) in the brain tissues, but the content of 5 -HT was decreased in the plasma, thus achieving dual regulation. The authors speculated that this might be associated with activating different 5-HT receptor subtypes, but explicit validation was not given. Crucially, no adverse effects were detected in vivo and in vitro. ZR-CDs supply a better green and safe analgesic strategy than opioids with side effects (respiratory suppression, drug dependence, etc.) [136].

HM-CDs have been investigated and documented from the above discussion to have considerable therapeutic 


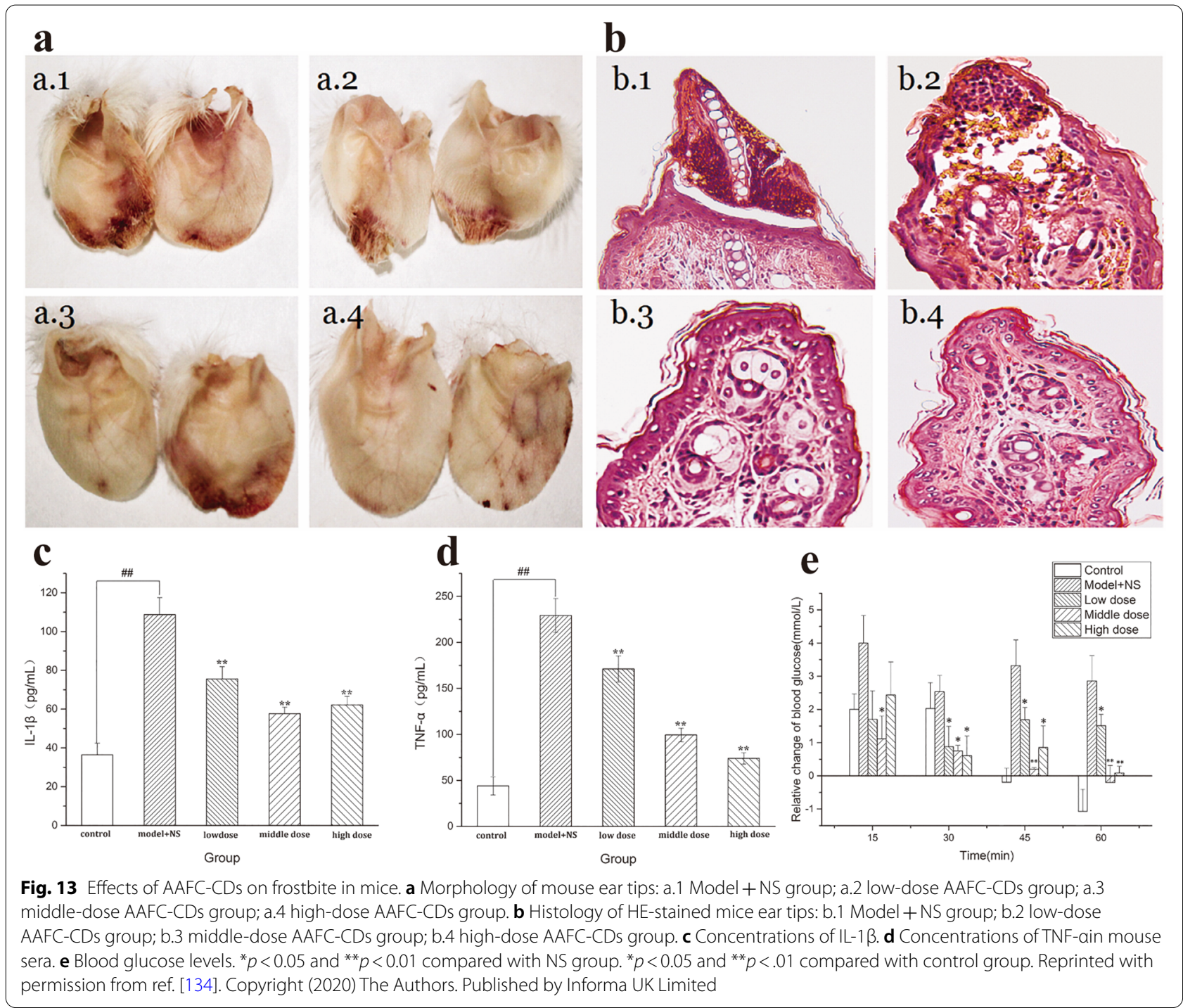

effects in many diseases. Nevertheless, three questions remain. (i) Components of HM-CDs are a mystery. Hightemperature conditions may cause the decomposition of herbal medicine, leading to reduction or even disappearance of active ingredients. The difference between HM-CDs and herbal medicine has not yet been clarified. (ii) Metabolic process in vivo is unknown. The essence of HM-CDs is nanoparticles, and the metabolic process is critical. Elucidating the metabolic mechanisms is one of the most notable challenges. (iii) Theranostic systems have not been realized. There is no report on HM-CDs as both an in vivo imaging agent and a therapeutic agent.

\section{Ion and molecule detection}

$\mathrm{Fe}^{3+}, \mathrm{Cu}^{2+}, \mathrm{Al}^{3+}$ and $\mathrm{Ag}^{+}$are abundant transition metal ions in biological systems. These ions play vital roles in many physiological and pathological processes, such as cell metabolism, cell proliferation, catalysis and DNA synthesis [137]. Some ions have been confirmed to fluctuate with the occurrence of a specific disease. For instance, aberrant fluctuation of $\mathrm{Fe}^{3+}$ is a marker of chronic heart failure [138]. However, excessive amounts of metal ions from ingestion of contaminated water and food can be highly toxic to organisms. Therefore, it is necessary to test the level of metal ions, such as $\mathrm{Cr}^{6+}$ and other toxic metal ions, in living organisms and everyday products. Nowadays, methods such as atomic absorption spectrometry [139], inductively coupled plasma mass spectrometry [140] and electrochemistry [141] have been utilized to detect metal ions. But these techniques are expensive and complex [80, 142]. A safe, efficient, sensitive and reliable means need to be developed. Owing to the high fluorescence stability, CDs play a unique strength in molecular and ion detection. When 
Table 3 HM-CDs as sensors

\begin{tabular}{|c|c|c|c|}
\hline Herbal medicine & Iron/molecule & Sensing mechanisms & Refs \\
\hline Jujubes & $\mathrm{Fe}^{3+}$ & Electron transfer & [149] \\
\hline Honey & $\mathrm{Fe}^{3+}$ & Electron transfer & [43] \\
\hline Lycii Fructus & $\mathrm{Fe}^{3+}$ & IFE/electron transfer/energy transfer & [148] \\
\hline Pinellia Ternata & $\mathrm{Cr}^{6+}$ & Synergistic effect of IFE and electron transfer & [151] \\
\hline Osmanthus fragrans & $\mathrm{Al}^{3+} /$ quercetin & IFE and static quenching effect & [152] \\
\hline Osmanthus fragrans & $\mathrm{Fe}^{3+}$ & IFE & [150] \\
\hline Mustard seeds & Ascorbic acid & Electron transfer & [157] \\
\hline Ginkgo leaf & Salazosulfapyridine & IFE & [85] \\
\hline Papaya & $\mathrm{Fe}^{3+} /$ Escherichia coli & Electron transfer/FimH proteins interact with mannose & [78] \\
\hline Purple perilla & $\mathrm{Ag}^{+}$ & Electron transfer & [142] \\
\hline Orange peel & $\mathrm{Cr}^{6+}$ & IFE & [84] \\
\hline Orange peel & Escherichia coli & Aptamer to E. coli cells & [103] \\
\hline Lychee seeds & Methylene blue & Electron transfer & [96] \\
\hline Rose flower & Tetracycline & Energy transfer & [153] \\
\hline Giant Knotweed Rhizome & $\mathrm{Hg}^{2+}$ & Energy transfer & {$[87]$} \\
\hline Bamboo leaves & $\mathrm{Cu}^{2+}$ & IFE & {$[80]$} \\
\hline Panax notoginseng & $\mathrm{Cr}^{6+}$ & IFE & [38] \\
\hline Hair & $\mathrm{Fe}^{3+} / \mathrm{ATP} / \mathrm{NAPDH}$ & $\begin{array}{l}\text { Synergistic action of the metabolism process by the digestive system } \\
\text { and quenching effect of internal metabolite and substance }\end{array}$ & [42] \\
\hline Yams & $\mathrm{Hg}^{2+} / 6$-mercaptopurine & FRET/decreased conjugation & {$[79]$} \\
\hline Gardenia fruit & $\mathrm{Hg}^{2+} /$ cysteine & Static quenching & [88] \\
\hline Coix seed & Furazolidone & Static quenching/IFE & {$[82]$} \\
\hline Smilax China & $\mathrm{Cu}^{2+}$ & Static quenching & {$[147]$} \\
\hline
\end{tabular}

IFE: inner filter effect; FRET: fluorescence resonance energy transfer

CDs combine with molecules or ions, their energy will be transferred, affecting the fluorescence properties (Table 3). Typically, the fluorescence quenching depends on several mechanisms, including the inner filter effect (IFE), fluorescence resonance energy transfer (FRET), static quenching effect, dynamic quenching effect and electron transfer [143-147]. This section discusses the application and sensing mechanisms of HM-CDs in ion and molecular detection (Fig. 14a).

\section{Detection of $\mathrm{Fe}^{3+}$}

Wang et al. [78] prepared two kinds of papaya-CDs while water and ethanol were used as the solvent (W-CDs and E-CDs) for selective $\mathrm{Fe}^{3+}$ detection. In different concentrations of $\mathrm{Fe}^{3+}$, the fluorescence emission of CDs at $450 \mathrm{~nm}$ decreased, which was since both CDs contain sufficient oxygen-containing functional groups $(-\mathrm{OH}$ and $-\mathrm{COOH})$ to form complexes with $\mathrm{Fe}^{3+}$. Moreover, the content of oxygen-containing functional groups in E-CD was higher than that in W-CD, so E-CD was more sensitive (detection limit, DL, was $0.29 \mu \mathrm{M})$. The detection performance was confirmed to be comparable with the Phenanthroline spectrophotometry by detecting iron in the heme capsules.
Another CD consistent with this fluorescence quenching is honey-CDs. Yang et al. [43] applied honey-CDs to detect $\mathrm{Fe}^{3+}$ based on the direct interactions between $\mathrm{Fe}^{3+}$ and $-\mathrm{COOH},-\mathrm{OH}$ and $-\mathrm{NH}_{2}$ (Fig. 14b, c). The DL of $\mathrm{Fe}^{3+}$ was $1.7 \times 10^{-9} \mathrm{~mol} / \mathrm{L}$ at a signal-to-noise ratio of three. The standard recovery experiments noted that the recoveries of human blood samples were $96.00 \%$, $97.25 \%$ and $101.50 \%$, which demonstrated the utility of honey-CDs [43].

Sun et al. [148] synthesized the water-soluble CDs with Lycii Fructus (LF-CDs). LF-CDs can selectively and sensitively detect $\mathrm{Fe}^{3+}$. The DL was estimated to be $21 \mathrm{nM}$. The absorbance intensity increased with $\mathrm{Fe}^{3+}$ concentrations, indicating that IFE might be one reason for fluorescence quenching. Additionally, LF-CDs chelated $\mathrm{Fe}^{3+}$ with abundant surface functional groups, such as $\mathrm{C}-\mathrm{OH}$ and $\mathrm{C}-\mathrm{O}-\mathrm{C}$. The complexes accelerated the nonradiative electron/hole recombination through effective photoelectron or energy transfer, resulting in further fluorescence quenching [148]. The fluorescence quenching of hair-derived CDs was consistent with LF-CDs. $\mathrm{Fe}^{3+}$ was also detected using other HM-CDs, such as CDs derived from jujubes via electron transfer [149], Osmanthus fragrans Lour-CDs via IFE [150]. 


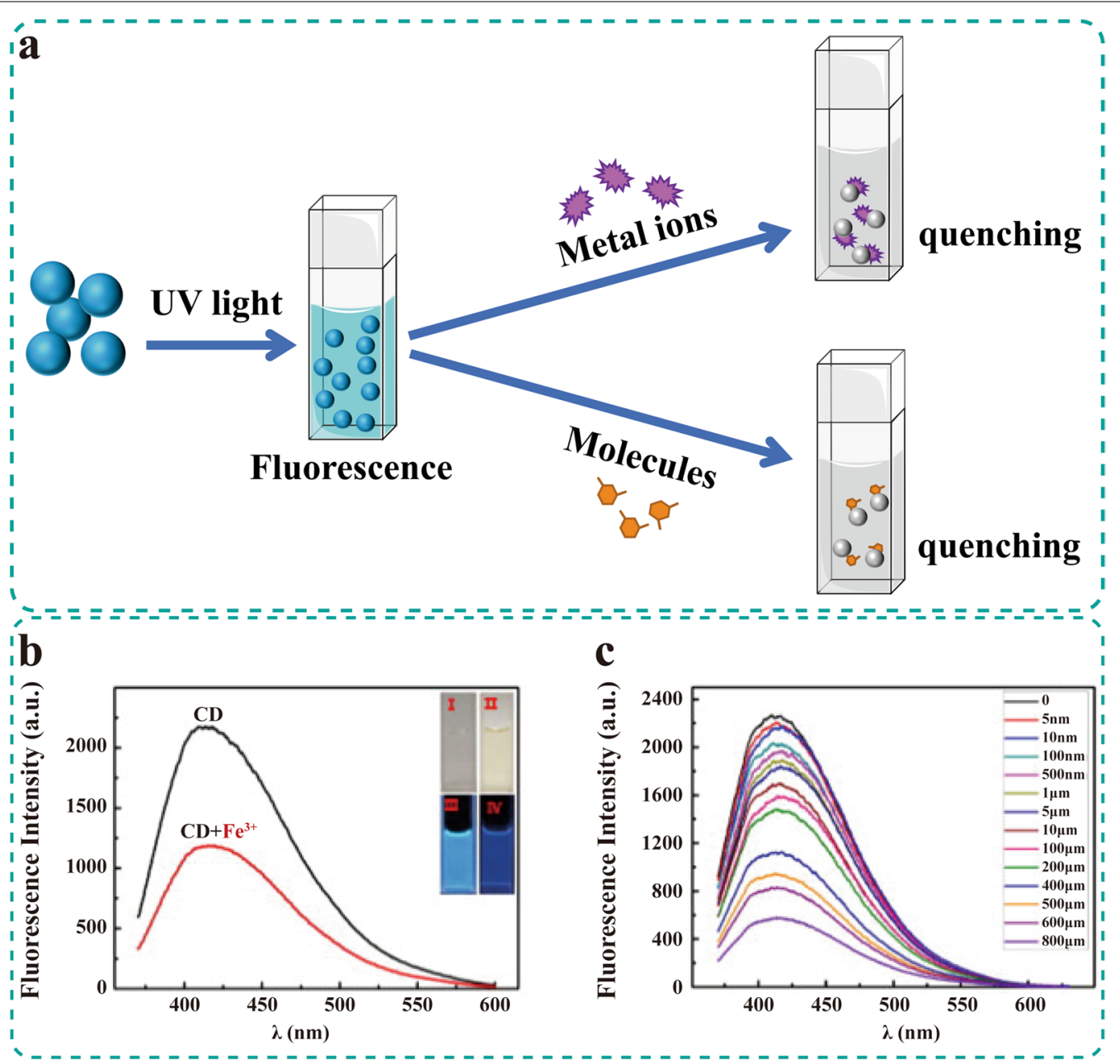

Fig. $14 \mathrm{HM}-\mathrm{CD}$ s as sensing agents. a Binding of metal ions or molecules on CDs causes fluorescence quenching. $\mathbf{b}$ Fluorescence spectra of honey-CDs in the absence (black) and presence (red) of $100 \mu \mathrm{M} \mathrm{Fe}^{3+}$. Inset: photographs. c Fluorescence spectra of honey-CDs in the presence of different concentrations of $\mathrm{Fe}^{3+}$. Reprinted with permission from ref. [43]. Copyright (2014) Elsevier

\section{Detection of $\mathrm{Cr}^{6+}$}

Dai et al. [151] used Pinellia ternata as a precursor and ethylenediamine as a nitrogen source to synthesize nitrogen-doped Pinellia quantum dots (PTN-CQDs). This CD detected $\mathrm{Cr}^{6+}$ selectively and sensitively in different water samples, and the DL was $15 \mathrm{nM}$. In addition to IFE, $\mathrm{Cr}^{6+}$ was easy to capture electrons because of the strong electron acceptance and affinity. As a result, when the excited state molecules of PTN-CQDs collided with $\mathrm{Cr}^{6+}$, it was highly prone to complexes, causing electron transfer and nonradiative transitions, leading to fluorescence quenching.

To ameliorate the selectivity of CDs, Wang et al. [84] used EDTA (a metal chelator) to modify an orange peel quantum dot (CDs@EDTA). They only found that IFE but not electron transfer was associated with fluorescence quenching. Furthermore, Panax notoginseng carbon dots (Pn-CDs) also detected $\mathrm{Cr}^{6+}$ via IFE, and the DL was $0.185 \mathrm{nM}$ [38].

\section{Detection of $\mathrm{Hg}^{2+}$}

$\mathrm{Li}$ et al. [79] used Chinese yams as a source of carbon and nitrogen to prepare water-soluble yam quantum dots and modified the surface with carboxyfluorescein (FAM)-DNA macro-molecules (NCDs-ssDNA) to promote strong $\pi-\pi$ accumulation. When 6-mercaptopurine (6-MP) was added, it could form a large conjugated rigid plane structure with FAM-DNA, resulting in fluorescence enhancement. However, the addition of $\mathrm{Hg}^{2+}$ terminated this reaction because the binding of $\mathrm{Hg}^{2+}$ to 
DNA disrupted the original system. This response pattern is called "on-off-on sensing mode."

Similarly, Sun et al. [88] produced the nitrogen and sulfur co-doped carbon dots (N/S-CDs) using gardenia fruit without any surface modifications. N/S-CDs were used to detect $\mathrm{Hg}^{2+}$ and cysteine. $\mathrm{Hg}^{2+}$ and $\mathrm{S}^{-}$could form $\mathrm{Hg}^{2+}$-S bonds, and caused fluorescence to decrease. Subsequently, due to the strong interaction between $\mathrm{Hg}^{2+}$ and the sulfhydryl group of cysteine, the $\mathrm{Hg}^{2+}{ }_{-} \mathrm{S}$ bond was destroyed after adding cysteine to the above system, thereby restoring fluorescence.

\section{Detection of $A /^{3+}$}

Yu et al. [152] used Osmanthus fragrans to synthesize nitrogen-doped quantum dots to detect quercetin and $\mathrm{Al}^{3+}$. When $\mathrm{Al}^{3+}$ was added to the $\mathrm{CDs}$ and quercetin system, it was easier to form complexes due to the strong binding force between $\mathrm{Al}^{3+}$ and quercetin, which naturally eliminated the IFE effect and restored fluorescence [152]. Meanwhile, the human bladder cancer T24 cells viability remained more than $90 \%$ when incubated at $1000 \mu \mathrm{g} / \mathrm{mL}$ CDs concentration. The CDs undoubtedly provide a green and safe means to detect $\mathrm{Al}^{3+}$ and quercetin in the biological field.

\section{Detection of $\mathrm{Cu}^{2+}$}

Liu et al. [80] synthesized CDs using bamboo leaves and modified them with branched polyethyleneimine (BPEI) with a robust chelating effect (BPEI-CDs). The BPEI$\mathrm{CDs}$ were used for detecting $\mathrm{Cu}^{2+}$ in environmental water. The DL was as low as $115 \mathrm{nM}$, and the dynamic range was $0.333-66.6 \mu \mathrm{M}$. The quenching principle was attributed to IFE of the copper amine complex formed at the surface of BPEI-CDs [80]. It offered a green, safe and high-efficient alternative approach for detecting $\mathrm{Cu}^{2+}$ in the water, especially industrial wastewater. Another work synthesized Smilax China-derived yellow-fluorescent CDs (y-CDs) by hydrothermal method [147]. Based on the static quenching effect, $\mathrm{Cu}^{2+}$ was highly selective detected (among 23 ions and 14 amino acids) with linear ranges of $0.5-10 \mu \mathrm{M}, 75-225 \mu \mathrm{M}$ and $250-350 \mu \mathrm{M}$, achieving a DL of $28 \mathrm{nM}$ [147].

\section{Detection of $\mathrm{Ag}^{+}$}

Zhao et al. [142] prepared purple perilla CDs with low toxicity and biocompatibility by hydrothermal synthesis. The CDs detected $\mathrm{Ag}^{+}$sensitively and selectively with a $\mathrm{DL}$ of $1.4 \mathrm{nM}$. Nonradiative electron transfer that occurs from the excited state to the $\mathrm{d}$ orbital of $\mathrm{Ag}^{+}$caused the fluorescence quenching. To obtain better sensing performances, this work optimized the experimental conditions, as follows. The $\mathrm{pH}$ was adjusted at 7.0, reaction time was $15 \mathrm{~min}$, the temperature was maintained at $30{ }^{\circ} \mathrm{C}$, and the concentration of CDs was $300 \mu \mathrm{g} / \mathrm{ml}$. Choosing CDs as fluorescence sensors requires constant exploration of optimal reaction conditions to ensure stable preparation in the future.

\section{Detection of molecules}

Due to the green and safety of herbal medicine, it is well suited to detect certain toxic substances in food, drinking water and other resources.

Detection of methylene blue Xue et al. [96] used CDs derived from lychee seeds (LS-CDs) to detect methylene blue $(\mathrm{MB})$ which could be adsorbed on the surface of LS-CDs, causing fluorescence quenching. LS-CDs are an encouraging means to detect toxic $\mathrm{MB}$ in wastewater because of low toxicity.

Detection of salazosulfapyridine Jiang et al. [85] produced nitrogen-doped quantum dots derived from ginkgo leaves to detect salazosulfapyridine (SASP). SASP could absorb the excitation of CDs to cause fluorescence quenching via IFE. Furthermore, the fluorescence lifetime of CDs was not related to SASP, which means that static quenching was also the possibility.

Detection of tetracycline CDs derived from rose flowers were adopted for detecting tetracycline via interaction [153]. This interaction resulted from the captured energy from CDs by forming new bonds with tetracycline, thus quenching fluorescence effectively.

Detection of furazolidone Furazolidone is a nitrofuran antibiotic but causes side reactions once excessive use. A highly fluorescent coix seeds $C D$ was prepared for detecting furazolidone with a linear range of $0.5-100 \mu \mathrm{M}$ [82]. The static quenching and IFE were possible quenching mechanisms [82]. As a novel fluorescence probe, coix seed-CD has simplicity, high selectivity and sensitivity relative to existing approaches (e.g., high-performance liquid chromatography, liquid chromatograph mass spectrometer, liquid chromatography with tandem mass spectrometry, electrochemical methods).

Although HM-CDs have been available for ion and molecule detection in some biological samples, other ions and molecules remain constantly explored. The water source is currently the primary sample tested by HM-CDs. Expanding the detection of substances in biological tissues using HM-CDs is highly warranted, such as blood, urine, saliva, tears and other liquid tissues convenient for collection. Other than that, it is also necessary to develop a sensing system for solid tissues. For example, detecting specific ions and molecules in brain tissue can rely on the BBB permeability of HM-CDs and the fluorescence quenching to achieve a safe and effective diagnosis and even treatment. 
Table 4 HM-CDs in bioimaging

\begin{tabular}{|c|c|c|c|}
\hline Herbal medicine & Organisms & Fluorescence color & Refs \\
\hline Honey & HepG2/HeLa cells & Green & [43] \\
\hline Lycii Fructus & HeLa cell & Blue/Glaucous/Green & [148] \\
\hline Lemon juice & Onion epidermal cell & Blue-green & [155] \\
\hline Ginkgo fruits & HeLa/KYSE410 cells & Blue/Green & {$[81]$} \\
\hline Lychee seeds & HepG2 cell & Blue & [96] \\
\hline Watermelon peel & HeLa cell & Green & [33] \\
\hline Ginger & MCF-10A/A549/MDA-MB-231/HeLa/HepG2 cells & Blue/Green/Red & [28] \\
\hline Panax notoginseng & $\begin{array}{l}\text { E. coli, B. cereus, S. cerevisiae/onion epidermal cells/leaf of } N \text {. } \\
\text { physaloides/BALB/C mice }\end{array}$ & Blue/Green/Red & [38] \\
\hline Osmanthus fragrans & T24 cell & Blue & {$[152]$} \\
\hline Osmanthus fragrans & A549 cell & Blue & [150] \\
\hline Mulberry silkworm cocoon & Zebrafish/embryos & Blue/Green/Red & {$[45]$} \\
\hline Hair & Zebrafish/embryos & Blue/Green/Red & [42] \\
\hline Coix seed & Furazolidone & Blue/Green & {$[82]$} \\
\hline Smilax China & PC12 cell & Blue/Yellow/Red & {$[147]$} \\
\hline Papaya & HeLa cell & Blue/Green & [78] \\
\hline Purple perilla & HeLa cell & Blue & [142] \\
\hline
\end{tabular}

\section{Bioimaging}

HM-CDs have better biocompatibility, availability and sustainability than chemical-CDs [23, 154], further extending the scope of biomedicine. In this section, we summarize the applications of HM-CDs in bioimaging (Table 4).

\section{Cellular imaging}

Due to the nanoscale size, HM-CDs are highly prone to be absorbed by cells, making them suitable for fluorescence imaging (Fig. 15) [154]. After labeling HeLa cells with LF-CDs, bright blue, glaucous and green luminescence were observed under the ultraviolet filter, $\mathrm{V}$ filter and B filter, respectively [148]. With the subsequent addition of $\mathrm{Fe}^{3+}$, the fluorescence intensity was decreased significantly. The lemon juice CDs were pursued as an imaging agent of onion epidermal cells [155]. The threedimensional structures of the cell wall and cell nucleus were visible, and no adverse effects on the organisms. After incubating HeLa and KYSE410 cells with H-NCDs for $8 \mathrm{~h}$, blue and green fluorescence were observed under excitation at $405 \mathrm{~nm}$ and $488 \mathrm{~nm}$, separately [81]. By comparing the fluorescence intensity of the nucleus and cytoplasm, researchers revealed that the CDs mainly entered into the cytoplasm. Although H-N-CDs did not cause cytotoxicity, whether fluorescence can discriminate different cells has not been confirmed.

In addition, Xue et al. [96] used LS-CDs to intervene in HepG2 cells. LS-CDs could penetrate cells and emit blue fluorescence to label cell membranes and cytoplasm. Other CDs used for imaging of HepG2 or HeLa cells also contained honey-CDs [43] and watermelon peel-CDs [33]. Of course, HM-CDs have been more versatile for other cell lineages imaging (Table 4). All above HM-CDs maintained the high viability of normal cells. However, researchers did not give us an indication of targeting of HM-CDs.

\section{Other imaging}

Next to cellular imaging, few HM-CDs were applied to other biological imaging. A classic example is nitrogendoped Pn-CDs [38], which was used for fluorescence imaging of multiple organisms. In microorganism imaging, Pn-CDs clearly labeled E. coli, B. cereus and S. cerevisiae. The blue, green and red fluorescence were observed at excitation wavelengths of 405, 488 and $543 \mathrm{~nm}$, respectively (Fig. 16a-c). As the size of the microorganism increased, the fluorescence intensity was also enhanced. Pn-CDs marked the cell membrane and nuclear membrane in protozoan imaging by explicitly identifying the cell membrane structure or phospholipid bilayer of P. caudatum (Fig. 16d-f). In plant imaging, Pn-CDs spread along the veins of Arabidopsis thaliana under the induction of transpiration. Finally, they realized the distribution of whole leaves by spreading in the intercellular space (Fig. 16g). In animal imaging, the fluorescence intensity of Pn-CDs in BALB/c mice first increased and then decreased with increasing time. Pn-CDs were distributed throughout the body through the bloodstream, gradually absorbed by the stomach, and finally excreted through the intestine and bladder (Fig. 16h). Most notably, there was fluorescence expression in the brain at $9 \mathrm{~h}$, 

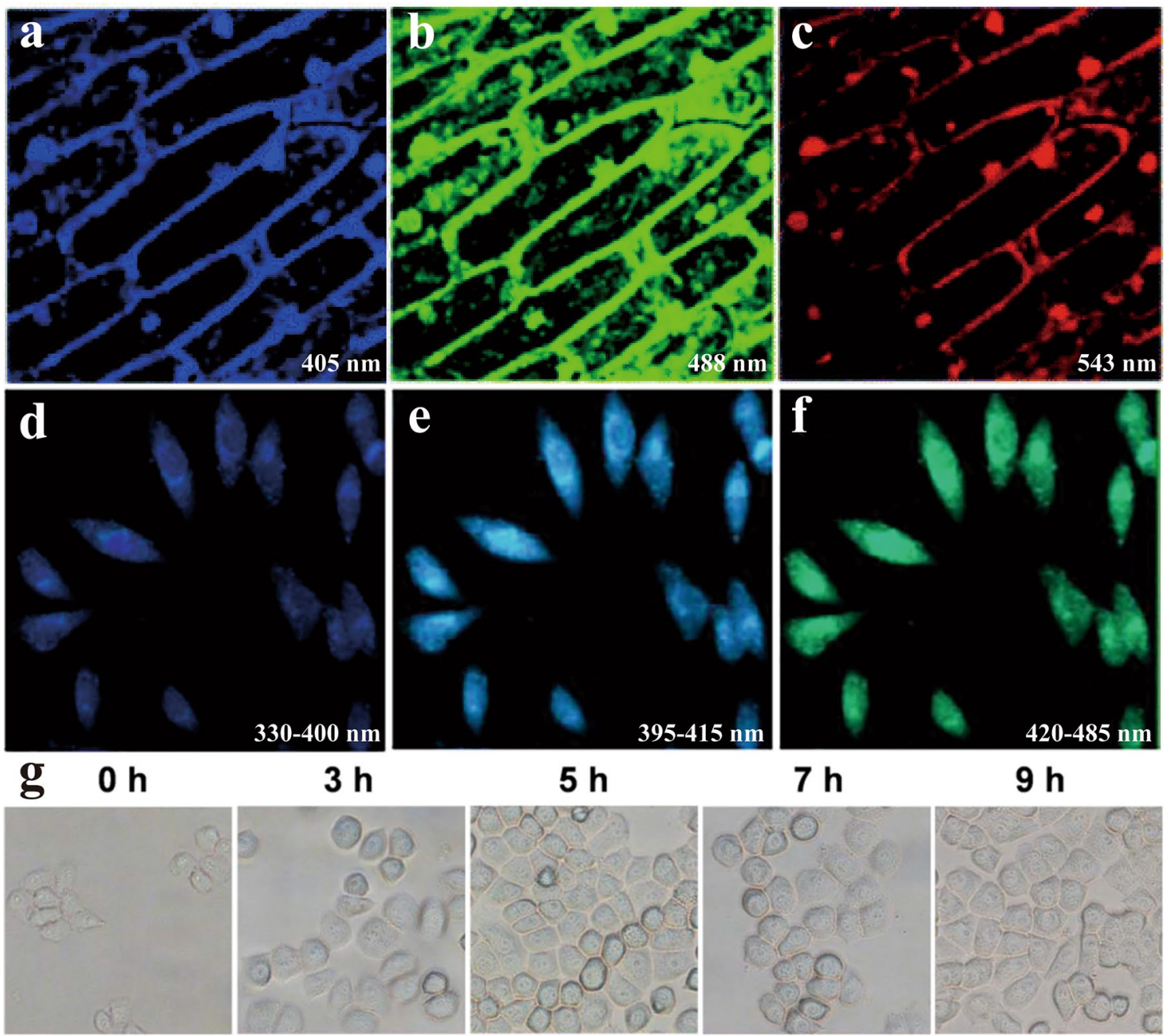

$3 \mathrm{~h}$

$5 \mathrm{~h}$
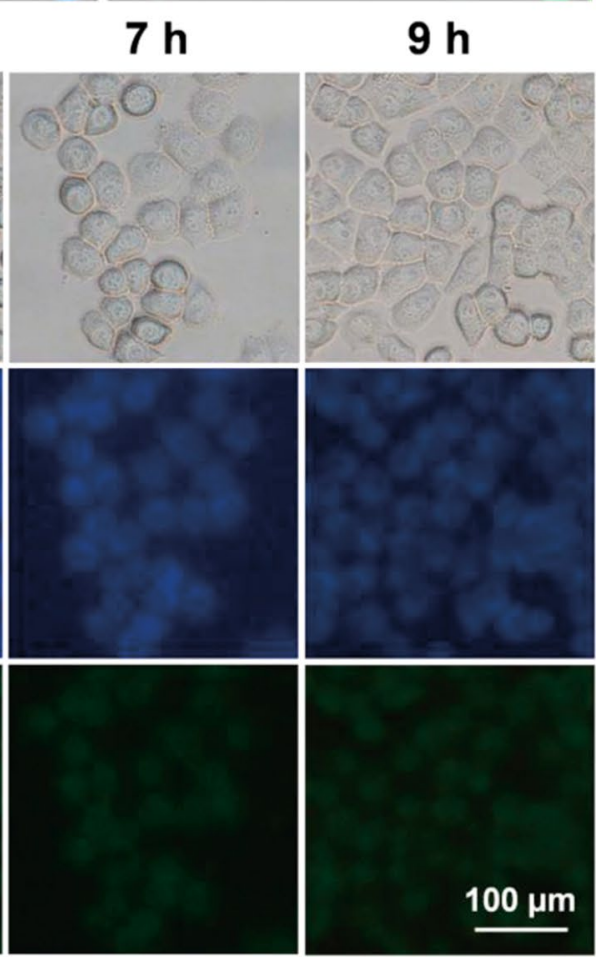

Fig. 15 Cellular imaging applications of HM-CDs. a-c CLSM image of onion skin cells with Pn N-CDs at a $405 \mathrm{~nm}$ laser, $488 \mathrm{~nm}$ laser, and $543 \mathrm{~nm}$ laser. Reprinted with permission from ref. [38]. Copyright (2020) Royal Society of Chemistry. d-f f Fluorescence microscope images of LF-CDs under UV-filter (330-400 nm), V-filter (395-415 nm) and B-filter (420-485 nm). Reprinted with permission from ref. [148]. Copyright (2017) Elsevier. $\mathbf{g}$ Fluorescence images of HeLa cells incubating with $1 \mathrm{mg} / \mathrm{mL}$ of W-CDs for $0 \mathrm{~h}, 3 \mathrm{~h}, 5 \mathrm{~h}, 7 \mathrm{~h}$ and $9 \mathrm{~h}$. 1st row: bright field images; 2nd row: the images with an excitation/emission wavelength at $340 \mathrm{~nm} / 420 \mathrm{~nm} ; 3 \mathrm{rd}$ row: the images with an excitation/emission wavelength at $495 \mathrm{~nm} / 520 \mathrm{~nm}$. Reprinted with permission from ref. [78]. Copyright (2016) Elsevier 

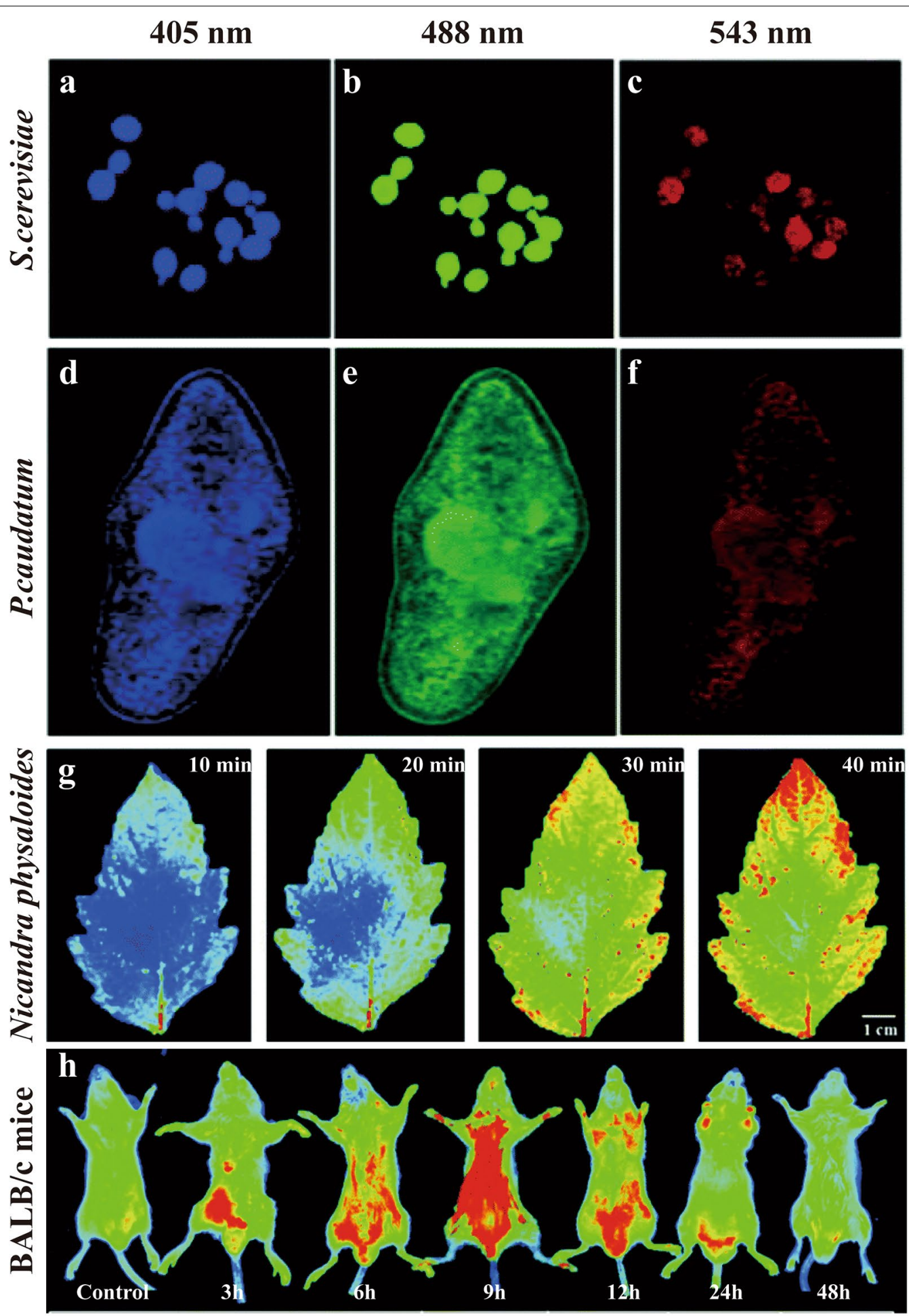

Fig. 16 Other imaging applications of HM-CDs. a-c CLSM images of S. cerevisiae incubated with Pn N-CDs at $405 \mathrm{~nm}, 488 \mathrm{~nm}, 543 \mathrm{~nm}$ laser, $\mathbf{d}-\mathbf{f}$ CLSM images of P. caudatum incubated with Pn N-CDs at $405 \mathrm{~nm}, 488 \mathrm{~nm}, 543 \mathrm{~nm}$ laser, $\mathbf{g}$ Imaging of N. physaloides with the injection of Pn N-CDs at different time intervals, $\mathbf{h}$ In vivo imaging of male BALB/C mice after injection of Pn N-CDs at different time intervals. Reprinted with permission from ref. [38]. Copyright (2020) Royal Society of Chemistry 


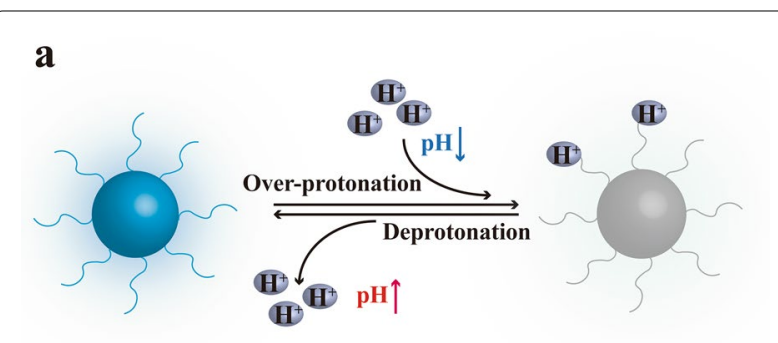

b

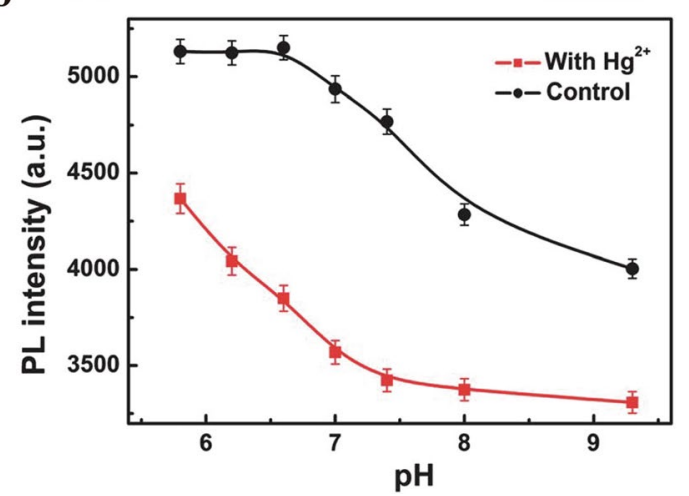

Fig. $17 \mathrm{pH}$ sensing of HM-CDs. a The response mechanism of $\mathrm{pH}$-sensitive $\mathrm{HM}-\mathrm{CDs}$, $\mathbf{b} \mathrm{pH}$ dependence for $\mathrm{Hg}^{2+}$ detection using photoluminescent $\mathrm{CDs}$ derived from giant knotweed rhizome. $c\left(\mathrm{Hg}^{2+}\right): 100.0$ mM; pH: 5.8, 6.2, 6.6, 7.0, 7.4, 8.0, 9.3; ex: 320 nm; em: $400 \mathrm{~nm}$. Each point is an average of three successive measurements. Reprinted with permission from ref. [87]. Copyright (2013) Royal Society of Chemistry

which means that Pn-CDs were able to penetrate the BBB. This performance is significantly better than macromolecules. Thus, HM-CDs may offer a novel mode of administration for herbal medicine to treat brain diseases.

Although HM-CDs are poorly studied in bioimaging, they will provide a safe and reliable novel strategy in the future. Exploiting the potential of HM-CDs in live imaging is necessary, mainly in disease-location, which will contribute to the successful construction of theranostics of HM-CDs.

\section{$\mathrm{pH}$ sensing}

Different diseases or body parts have various $\mathrm{pH}$ values [156]. Some of the HM-CDs have shown superior $\mathrm{pH}$-responsive properties (Fig. 17), making them more suitable for different $\mathrm{pH}$ conditions. BPEI-CDs derived from bamboo leaves demonstrated a $\mathrm{pH}$-dependent increase in fluorescence intensity in the $\mathrm{pH}$ range of 2-4 and decreased as $\mathrm{pH}$ further increased [80]. In a highly acidic $(\mathrm{pH}<4)$ solution, the over-protonation of PEI led to diminished fluorescence, but the opposite was true at $\mathrm{pH}>4$ due to the deprotonation of PEI. This phenomenon was caused by the formation of intramolecular or intermolecular hydrogen bonds by functional groups on the surface of $\mathrm{HM}-\mathrm{CDs}$, such as $\mathrm{N}-\mathrm{H}$ and $\mathrm{O}-\mathrm{H}$. Besides, CDs derived from fennel seeds [94], giant knotweed rhizome [87], pinellia ternata [151], mustard seeds [157], papaya [78] and Purple perilla [142] were also sensitive primarily to $\mathrm{pH}$. Within these HM-CDs, giant knotweed rhizome-CDs efficiently detected $\mathrm{Hg}^{2+}$ based on photoluminescent from pH 5.8 to 9.3 (Fig. 17) [87] owing to the hydroxyl and carboxylic groups. It signifies that giant knotweed rhizome-CDs warrant as an $\mathrm{Hg}^{2+}$ fluorescent nanoprobe in either an acidic or alkaline medium.

\section{Conclusions and outlook}

This review firstly systematically summarizes the research advances on HM-CDs by focusing on their synthetic approaches and specific applications. As a new branch of CDs, HM-CDs have been used in disease treatment, ion and molecular detection, bioimaging and $\mathrm{pH}$ sensing. The potential therapeutic effect is a meaningful sign that differentiates HM-CDs from other CDs. The medicinal value of herbal medicine may cause HM-CDs to contain medicinal substances without loading drugs, that may effectively avoid harmful effects. Furthermore, the application of HM-CDs in bioimaging has laid a solid foundation for theranostics. We expect that HM-CDs will make significant progress in the near future. Besides, the following questions also need to be addressed:

More efficient and stable synthesis Currently, two most common approaches for synthesizing HM-CDs are hydrothermal synthesis and high-temperature pyrolysis. Even though this "one-key" synthesis broadens the scope of HM-CDs' applications, the QY, particle size, and fluorescence intensity are not stable. A more convenient and efficient microwave method is gradually appreciated which not only guarantees ecology and safety, but also enhances the efficiency of synthesis. More efficient and stable approaches require further development for efficient synthesis in the future.

Identification of active ingredients Although HM-CDs have unique advantages for therapy, their intrinsic effective ingredients are still uncertain. Active ingredients are essential to treat diseases. Identification of the effective substances is the direct evidence to elucidate mechanisms of action of HM-CDs. Existing synthesis methods may cause the decomposition or even induce disappearance of some practical components of herbal medicine at high temperatures. Therefore, remaining moieties of active compounds on HM-CDs under different synthesis methods and conditions is an important research direction in the future. Meanwhile, establishing an identification way to explore pharmacodynamic constituents of HM-CDs is the key to the subsequent research. At present, an effective strategy may be the high-performance 
liquid chromatography tandem mass spectrometry (HPLC-MS), which can be used as an available means for identifying pharmacodynamic constituents of HM-CDs.

Clarifications of metabolism and distribution in vivo The metabolism and distribution of nanomaterials have always been a hot issue. Although HM-CDs have shown excellent safety at the cellular level, studies on metabolism and distribution are still scarce. In vitro toxicity tests are far from sufficient to prove the safety of HM-CDs throughout the biological system. Future studies should manage to answer the following four questions: (i) What are HM-CDs' metabolic pathways in vivo? (ii) How are
HM-CDs distributed in vivo? (iii) Will different herbal precursors be accompanied by different metabolism and distribution in vivo? (iiii) After receiving long-term treatment, whether HM-CDs deposit in vivo and cause chronic toxicity? More complete in vivo toxicity tests, distribution and metabolism in vivo need to preferably reveal the unique functions of HM-CDs in organisms.

Realization of theranostics The photoluminescence of CDs is one of the most convincing characteristics. Research on the integration of diagnosis and treatment of HM-CDs seems boundless owing to the therapeutic effects. According to existing studies, the therapeutic

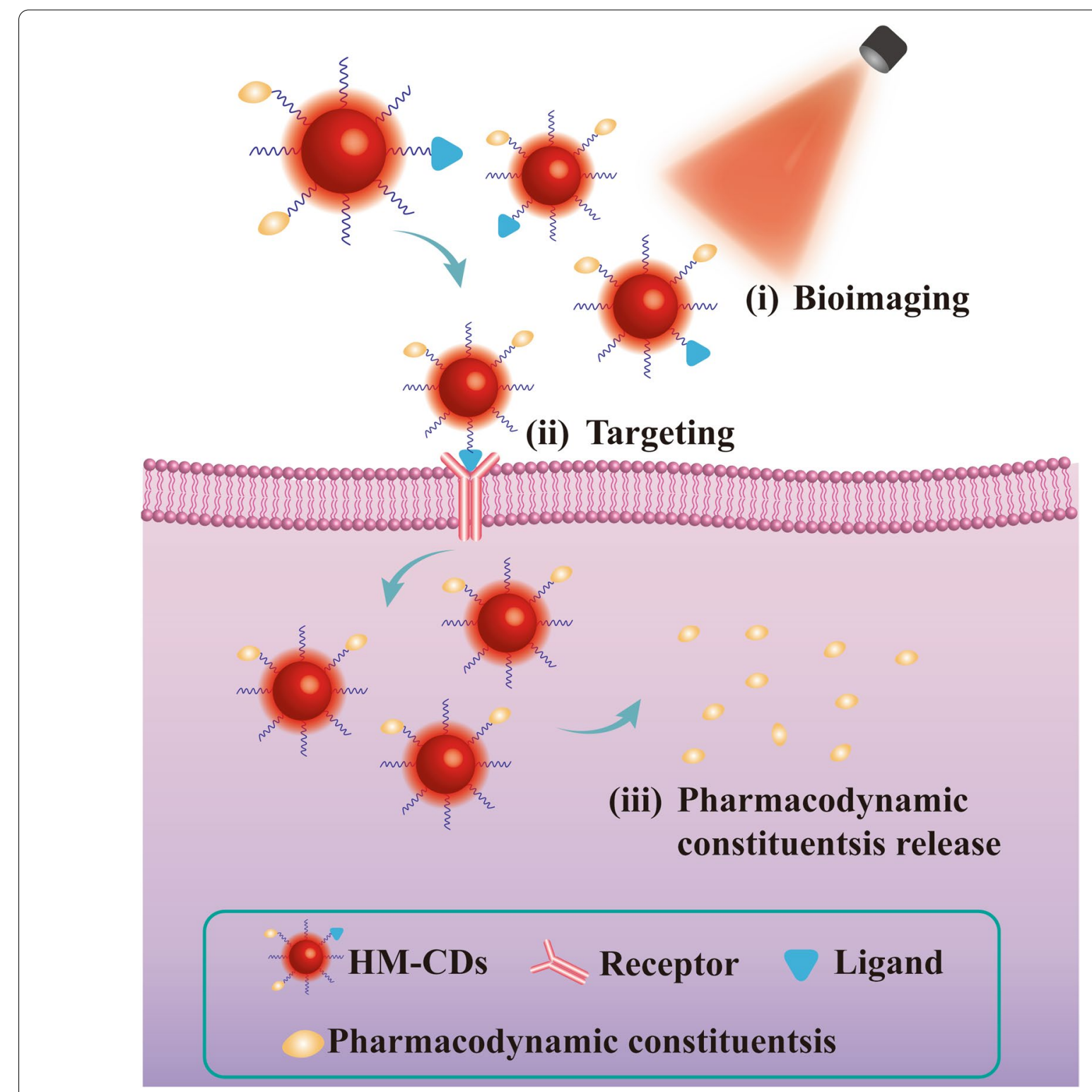

Fig. 18 The future theranostics strategies in the field of HM-CDs 
effects and bioimaging are separate from each other. Moreover, the investigation of bioimaging remains at a very preliminary stage. The combination of therapy and bioimaging will provide unlimited possibilities for realizing theranostics which becomes a huge challenge: (i) The premise of safety and simplicity are the guarantees of optimal targeted therapy of HM-CDs. (ii) The red-emission CDs exhibit little damage to the biological matrix, deep tissue penetration and minimum autofluorescence background of biosamples [158]. Therefore, synthesizing red HM-CDs is required for bioimaging applications stably and efficiently. (Fig. 18) (iii) Aberrant mitochondrial function is involved in a range of human diseases [159]. Thus, the imaging in mitochondria of HM-CDs is also the sharp focus in the future.

Exploration of different kinds of herbal medicine Phytomedicines are currently the main precursors of HMCDs. In addition, protein-rich medicines from animal drugs can act as sources of abundant heteroatoms or functional groups, which may help improve properties of HM-CDs. Therefore, CDs derived from animal drugs are a crucial area for future exploration.

Reductions of toxicity and increasing efficiency The minimum toxicity of HM-CDs triggered our consideration: Can HM-CDs achieve the purpose of reducing toxicity and increasing efficiency of toxic herbal medicine (such as manchurian dutchmanspipe stem, common monkshood, aristolochic acid)? If toxic reduction can be achieved, will the active ingredients change in the synthesis procedure, losing the original efficacy? Assuming it is feasible, to make toxic herbal medicine into CDs should be a novel processing tool. The solution to this issue will immensely drive the clinical applications of toxic herbal medicine.

Recycle of herbal residues The extraction efficiency of herbal medicine is approximately 50\% [160]. The residual herbal residues also contain some vital active ingredients. Strategies to recycle herbal residues are underway. In the future, we must attempt to extract CDs from herbal residues, and reuse them in the diagnosis and treatment of diseases, achieving optimal utilization of herbal medicine.

\footnotetext{
Abbreviations

HM-CDs: Herbal medicine-carbon dots; CDs: Carbon dots; JSX: Jiaosanxian; H-N-CD: Nitrogen-doped CDs from ginkgo fruits; $Q Y$ : Quantum yield; BBB: Blood-brain barrier; SHC-CDs: Schizonepetae Herba Carbonisata-CDs; H-CDs: Ginkgo fruits-CDs using hydrothermal methods; M-CDs: Ginkgo fruits-CDs using microwave methods; E-CDs: Ethanol-papaya CDs; W-CDs: Waterpapaya CDs; PTC-CDs: Pollen Typhae Carbonisata-CDs; APTT: Activated partial thromboplastin time; FIB: Fibrinogen; JMC-CDs: Junci Medulla CarbonisataCDs; EYO-CDs: Egg yolk oil-CDs; PCC-CDs: Phellodendri Cortex Carbonisatuscarbon dots; MSC-CDs: Mulberry silkworm cocoon-derived carbon dots; LJFC-CDs: Lonicerae japonicae Flos-derived carbon dots; AFIC-CDs: Aurantii fructus immaturus carbonisata-derived carbon dots; RSFC: Radix Sophorae
}

Flavescentis carbonisata; PLR-CDs: Puerariae lobatae Radix CDs; XOD: Xanthine oxidase; PRAC-CDs: Paeoniae Radix Alba Carbonisata-derived carbon dots; ALT: Alanine transaminase; AST: Acetone transaminase; TBA: Total bile acid; TBIL: Total bilium; MDA: Malondialdehyde; SOD: Superoxide dismutase; SCR: Serum creatinine; BUN: Blood urea nitrogen; UTP: Urine total protein; MALB: Microalbuminuria; AAFC-CDs: Artemisiae Argyi Folium Carbonisata-carbon dots; ZR: Zingiberis Rhizoma; ENK: Enkephalin; 5-HT: Serotonin; IFE: Inner filter effect; FRET: Fluorescence resonance energy transfer; DL: Detection limit; LF-CDs: Lycii Fructus-CDs; PTN-CQDs: Nitrogen-doped Pinellia quantum dots; Pn-CDs: Panax notoginseng carbon dots; FAM: Carboxyfluorescein; 6-MP: 6-Mercaptopurine; N/S-CDs: Nitrogen and sulfur co-doped carbon dots; y-CDs: Smilax China-derived yellow-fluorescent CDs; BPEI-CDs: Branched polyethyleneimine-CDs; LS-CDs: CDs derived from lychee seeds; MB: Methylene blue; SASP: Salazosulfapyridine.

\section{Acknowledgements}

Not applicable.

\section{Authors' contributions}

YWang brought forward the subject and guided the writing. WKL wrote the manuscript with the help of LLZ, ZYY and YWu. WZ, JKL and TT checked all sections of the manuscript. YWang, WKL and XHG made a revision. All authors read and approved the final manuscript.

\section{Funding}

This work was supported by the Project of Inheritance Studio for National Famous Experts of Traditional Chinese Medicine (No. [2018]134), the Fundamental Research Funds for the Central Universities of Central South University (No. 2021zzts1028), and the Innovation-Driven Project of Central South University (No. 2020CX047).

\section{Availability of data and materials}

Not applicable.

\section{Declarations}

\section{Consent for publication}

We have included 11 figures (Figs. 3, 4, 7, 9, 11, 12, 13, 14, 15, 16, 17) from previously published literature with required copyright permission. We have mentioned this in the manuscript with proper citation.

\section{Competing interests}

The authors report no competing interests in this work.

\section{Author details}

${ }^{1}$ Institute of Integrative Medicine, Department of Integrated Chinese and Western Medicine, Xiangya Hospital Central South University, Changsha, China. ${ }^{2}$ Hunan University of Chinese Medicine, Changsha, China. ${ }^{3}$ The College of Integrated Traditional Chinese and Western Medicine, Hunan University of Chinese Medicine, Changsha, China.

Received: 1 July 2021 Accepted: 30 September 2021

Published online: 13 October 2021

\footnotetext{
References

1. Xu X, Ray R, Gu Y, Ploehn HJ, Gearheart L, Raker K, Scrivens WA. Electrophoretic analysis and purification of fluorescent single-walled carbon nanotube fragments. J Am Chem Soc. 2004;126(40):12736-7.

2. Caglayan MO, Mindivan F, Sahin S. Sensor and bioimaging studies based on carbon quantum dots: the green chemistry approach. Crit Rev Anal Chem. 2020. https://doi.org/10.1080/10408347.2020.1828029.

3. Zheng M, Liu S, Li J, Qu D, Zhao H, Guan X, Hu X, Xie Z, Jing X, Sun Z. Integrating oxaliplatin with highly luminescent carbon dots: an unprecedented theranostic agent for personalized medicine. Adv Mater. 2014;26(21):3554-60.

4. Kang Z, Lee ST. Carbon dots: advances in nanocarbon applications. Nanoscale. 2019;11(41):19214-24.
} 
5. Du J, Xu N, Fan J, Sun W, Peng X. Carbon dots for in vivo bioimaging and theranostics. Small. 2019;15(32):e1805087.

6. Atabaev TS. Doped carbon dots for sensing and bioimaging applications: a minireview. Nanomaterials. 2018;8(5):342.

7. Devi P, Saini S, Kim KH. The advanced role of carbon quantum dots in nanomedical applications. Biosens Bioelectron. 2019;141:111158.

8. Loczechin A, Seron K, Barras A, Giovanelli E, Belouzard S, Chen YT, Metzler-Nolte N, Boukherroub R, Dubuisson J, Szunerits S. Functional carbon quantum dots as medical countermeasures to human coronavirus. ACS Appl Mater Interfaces. 2019;11 (46):42964-74.

9. Wang D, Liu L, Jiang J, Chen L, Zhao J. Polyoxometalate-based composite materials in electrochemistry: state-of-the-art progress and future outlook. Nanoscale. 2020;12(10):5705-18.

10. Pan M, Xie X, Liu K, Yang J, Hong L, Wang S. Fluorescent carbon quantum dots-synthesis, functionalization and sensing application in food analysis. Nanomaterials. 2020;10(5):930.

11. Shi X, Wei W, Fu Z, Gao W, Zhang C, Zhao Q, Deng F, Lu X. Review on carbon dots in food safety applications. Talanta. 2019;194:809-21.

12. Huang $P$, Lin J, Wang $X$, Wang Z, Zhang $C$, He M, Wang $K$, Chen F, Li Z, Shen G, Cui D, Chen X. Light-triggered theranostics based on photosensitizer-conjugated carbon dots for simultaneous enhancedfluorescence imaging and photodynamic therapy. Adv Mater. 2012;24(37):5104-10.

13. Goh EJ, Kim KS, Kim YR, Jung HS, Beack S, Kong WH, Scarcelli G, Yun SH, Hahn SK. Bioimaging of hyaluronic acid derivatives using nanosized carbon dots. Biomacromol. 2012;13(8):2554-61.

14. Liu R, Wu D, Liu S, Koynov K, Knoll W, Li Q. An aqueous route to multicolor photoluminescent carbon dots using silica spheres as carriers. Angew Chem Int Ed Engl. 2009;48(25):4598-601.

15. Lommens P, Lambert K, Loncke F, De Muynck D, Balkan T, Vanhaecke $\mathrm{F}$, Vrielinck $\mathrm{H}$, Callens $\mathrm{F}$, Hens Z. The growth of $\mathrm{Co}: \mathrm{ZnO} / \mathrm{ZnO}$ core/shell colloidal quantum dots: changes in nanocrystal size, concentration and dopant coordination. ChemPhysChem. 2008:9(3):484-91.

16. Wang C, Zhao T, Wang G, Gao J, Fang H. Superplastic forming and diffusion bonding of Ti-22Al-24Nb alloy. J Mater Process Technol. 2015;222:122-7.

17. Li X, Wang H, Shimizu Y, Pyatenko A, Kawaguchi K, Koshizaki N. Preparation of carbon quantum dots with tunable photoluminescence by rapid laser passivation in ordinary organic solvents. Chem Commun 2011:47(3):932-4.

18. Wang $Y$, Tang M. Review of in vitro toxicological research of quantum dot and potentially involved mechanisms. Sci Total Environ. 2018;625:940-62.

19. Kumar R, Kumar VB, Gedanken A. Sonochemical synthesis of carbon dots, mechanism, effect of parameters, and catalytic, energy, biomedical and tissue engineering applications. Ultrason Sonochem. 2020;64:105009.

20. Radnia F, Mohajeri N, Zarghami N. New insight into the engineering of green carbon dots: possible applications in emerging cancer theranostics. Talanta. 2020;209:120547.

21. Ansari L, Hallaj S, Hallaj T, Amjadi M. Doped-carbon dots: Recent advances in their biosensing, bioimaging and therapy applications. Colloids Surf B Biointerfaces. 2021:203:111743.

22. Zhang X, Jiang M, Niu N, Chen Z, Li S, Liu S, Li J. Natural-productderived carbon dots: from natural products to functional materials. Chemsuschem. 2018;11(1):11-24.

23. Tejwan N, Saha SK, Das J. Multifaceted applications of green carbon dots synthesized from renewable sources. Adv Colloid Interface Sci. 2020;275:102046

24. Cui F, Ye Y, Ping J, Sun X. Carbon dots: Current advances in pathogenic bacteria monitoring and prospect applications. Biosens Bioelectron. 2020:156:112085.

25. Dong X, Liang W, Meziani MJ, Sun YP, Yang L. Carbon dots as potent antimicrobial agents. Theranostics. 2020;10(2):671-86.

26. Ashrafizadeh M, Mohammadinejad R, Kailasa SK, Ahmadi Z, Afshar EG, Pardakhty A. Carbon dots as versatile nanoarchitectures for the treatment of neurological disorders and their theranostic applications: a review. Adv Colloid Interface Sci. 2020;278:102123.

27. Anand A, Unnikrishnan B, Wei SC, Chou CP, Zhang LZ, Huang CC. Graphene oxide and carbon dots as broad-spectrum antimicrobial agents_a mini review. Nanoscale Horiz. 2019;4(1):117-37.
28. Li CL, Ou CM, Huang CC, Wu WC, Chen YP, Lin TE, Ho LC, Wang CW, Shih CC, Zhou HC, Lee YC, Tzeng WF, Chiou TJ, Chu ST, Cang J, Chang HT. Carbon dots prepared from ginger exhibiting efficient inhibition of human hepatocellular carcinoma cells. J Mater Chem B. 2014;2(28):4564-71.

29. Li X, Zhang $Y$, Wang $Y, X u$ J, Xin $P$, Meng $Y$, Wang Q, Kuang $H$. The mechanisms of traditional chinese medicine underlying the prevention and treatment of Parkinson's disease. Front Pharmacol. 2017;8:634.

30. Ma Y, Chen M, Guo Y, Liu J, Chen W, Guan M, Wang Y, Zhao X, Wang X, Li H, Meng L, Wen Y, Wang Y. Prevention and treatment of infectious diseases by traditional Chinese medicine: a commentary. APMIS. 2019;127(5):372-84.

31. Teschke R, Wolff A, Frenzel C, Eickhoff A, Schulze J. Herbal traditional Chinese medicine and its evidence base in gastrointestinal disorders. World J Gastroenterol. 2015;21(15):4466-90.

32. Li FS, Weng JK. Demystifying traditional herbal medicine with modern approach. Nat Plants. 2017;3:17109.

33. Zhou J, Sheng Z, Han H, Zou M, Li C. Facile synthesis of fluorescent carbon dots using watermelon peel as a carbon source. Mater Lett. 2012;66(1):222-4.

34. Becraft AR, Sturm ML, Mendez RL, Park SH, Lee SI, Shay NF. Intake of watermelon or its byproducts alters glucose metabolism, the microbiome, and hepatic proinflammatory metabolites in high-fatfed male C57BL/6 J mice. J Nutr. 2020;150(3):434-42.

35. Li DC, Zhong XK, Zeng ZP, Jiang JG, Li L, Zhao MM, Yang XQ, Chen J, Zhang BS, Zhao QZ, Xie MY, Xiong H, Deng ZY, Zhang XM, Xu SY, Gao YX. Application of targeted drug delivery system in Chinese medicine. J Control Release. 2009;138(2):103-12.

36. Wang L, Zheng S, Huang G, Sun J, Pan Y, Si Y, Tu P, Xu G, Ma Y, Guo Y. Osthole-loaded N-octyl-O-sulfonyl chitosan micelles (NSC-OST) inhibits RANKL-induced osteoclastogenesis and prevents ovariectomy-induced bone loss in rats. J Cell Mol Med. 2020;24(7):4105-17.

37. Csermely P, Agoston V, Pongor S. The efficiency of multi-target drugs: the network approach might help drug design. Trends Pharmacol Sci. 2005;26(4):178-82.

38. Zheng X, Qin K, He L, Ding Y, Luo Q, Zhang C, Cui X, Tan Y, Li L, Wei Y Novel fluorescent nitrogen-doped carbon dots derived from Panax notoginseng for bioimaging and high selectivity detection of $\mathrm{Cr}(6)$. Analyst. 2020;146(3):911-9.

39. Kim JH, Doh EJ, Lee G. quantitative comparison of the marker compounds in different medicinal parts of Morus alba L. using high-performance liquid chromatography-diode array detector with chemometric analysis. Molecules. 2020;25(23):5592

40. Xiong A, Shao Y, Fang L, Yang X, Zhang S, Zheng J, Ding W, Yang $L$, Wang Z. Comparative analysis of toxic components in different medicinal parts of Gynura japonica and its toxicity assessment on mice. Phytomedicine. 2019;54:77-88.

41. Zhu L, Wang X, Li S, Qi ER, Meng J, Ching Lam KY, Dong X, Xu J, Chen $\mathrm{H}$, Zhao Z. Qualitative and quantitative characterization of carbohydrate profiles in three different parts of Poria cocos. J Pharm Biomed Anal. 2020;179:113009.

42. Zhang JH, Niu A, Li J, Fu JW, Xu Q, Pei DS. In vivo characterization of hair and skin derived carbon quantum dots with high quantum yield as long-term bioprobes in zebrafish. Sci Rep. 2016;6:37860.

43. Yang X, Zhuo Y, Zhu S, Luo Y, Feng Y, Dou Y. Novel and green synthesis of high-fluorescent carbon dots originated from honey for sensing and imaging. Biosens Bioelectron. 2014;60:292-8.

44. Zhao $Y$, Zhang $Y$, Liu X, Kong $H$, Wang $Y$, Qin $G$, Cao P, Song $X$, Yan $X$, Wang Q, Qu H. Novel carbon quantum dots from egg yolk oil and their haemostatic effects. Sci Rep. 2017;7(1):4452.

45. Wang X, Zhang Y, Kong H, Cheng J, Zhang M, Sun Z, Wang S, Liu J, Qu $\mathrm{H}$, Zhao Y. Novel mulberry silkworm cocoon-derived carbon dots and their anti-inflammatory properties. Artif Cells Nanomed Biotechnol. 2020;48(1):68-76.

46. Xia C, Zhu S, Feng T, Yang M, Yang B. Evolution and synthesis of carbon dots: from carbon dots to carbonized polymer dots. Adv Sci. 2019;6(23):1901316.

47. Yan Y, Chen J, Li N, Tian J, Li K, Jiang J, Liu J, Tian Q, Chen P. Systematic bandgap engineering of graphene quantum dots and applications 
for photocatalytic water splitting and $\mathrm{CO} 2$ reduction. ACS Nano. 2018;12(4):3523-32.

48. Zhi B, Yao X, Cui Y, Orr G, Haynes CL. Synthesis, applications and potential photoluminescence mechanism of spectrally tunable carbon dots. Nanoscale. 2019;11(43):20411-28.

49. Wang Z, Yuan F, Li X, Li Y, Zhong H, Fan L, Yang S. 53\% Efficient red emissive carbon quantum dots for high color rendering and stable warm white-light-emitting diodes. Adv Mater. 2017. https://doi.org/10.1002/ adma.201702910.

50. Yan F, Sun Z, Zhang H, Sun X, Jiang Y, Bai Z. The fluorescence mechanism of carbon dots, and methods for tuning their emission color: a review. Mikrochim Acta. 2019;186(8):583.

51. Wang X, Cheng Z, Zhou Y, Tammina SK, Yang Y. A double carbon dot system composed of N, Cl-doped carbon dots and N, Cu-doped carbon dots as peroxidase mimics and as fluorescent probes for the determination of hydroquinone by fluorescence. Mikrochim Acta. 2020;187(6):350.

52. Wang C, Wang Y, Shi H, Yan Y, Liu E, Hu X, Fan J. A strong blue fluorescent nanoprobe for highly sensitive and selective detection of mercury(II) based on sulfur doped carbon quantum dots. Mater Chem Phys. 2019;232:145-51.

53. Pal A, Ahmad K, Dutta D, Chattopadhyay A. Boron Doped carbon dots with unusually high photoluminescence quantum yield for ratiometric intracellular pH sensing. ChemPhysChem. 2019;20(8):1018-27.

54. Tejwan N, Saini AK, Sharma A, Singh TA, Kumar N, Das J. Metal-doped and hybrid carbon dots: a comprehensive review on their synthesis and biomedical applications. J Control Release. 2020;330:132-50.

55. Li F, Li T, Sun C, Xia J, Jiao Y, Xu H. Selenium-doped carbon quantum dots for free-radical scavenging. Angew Chem Int Ed Engl. 2017;56(33):9910-4.

56. Li F, Yang D, Xu H. Non-metal-heteroatom-doped carbon dots: synthesis and properties. Chemistry. 2019;25(5):1165-76.

57. Mohapatra S, Das RK. Dopamine integrated B, N, S doped CQD nanoprobe for rapid and selective detection of fluoride ion. Anal Chim Acta. 2019;1058:146-54.

58. Gong Y, Yu B, Yang W, Zhang X. Phosphorus, and nitrogen co-doped carbon dots as a fluorescent probe for real-time measurement of reactive oxygen and nitrogen species inside macrophages. Biosens Bioelectron. 2016;79:822-8.

59. Miao X, Yan X, Qu D, Li D, Tao FF, Sun Z. Red Emissive sulfur, nitrogen codoped carbon dots and their application in ion detection and theraonostics. ACS Appl Mater Interfaces. 2017;9(22):18549-56.

60. Dhenadhayalan N, Lin KC, Saleh TA. Recent advances in functionalized carbon dots toward the design of efficient materials for sensing and catalysis applications. Small. 2020;16(1):e1905767.

61. Yu Y, Shen M, Song Q, Xie J. Biological activities and pharmaceutical applications of polysaccharide from natural resources: a review. Carbohydr Polym. 2018;183:91-101.

62. Hu M, Yu Z, Wang J, Fan W, Liu Y, Li J, Xiao H, Li Y, Peng W, Wu C. Traditional uses, origins, chemistry and pharmacology of Bombyx batryticatus: a review. Molecules. 2017:22(10):1779.

63. Huie CW, Di X. Chromatographic and electrophoretic methods for Lingzhi pharmacologically active components. J Chromatogr B Analyt Technol Biomed Life Sci. 2004;812(1-2):241-57.

64. Wang $X$, Feng $Y$, Dong $P$, Huang J. A mini review on carbon quantum dots: preparation, properties, and electrocatalytic application. Front Chem. 2019;7:671

65. Tuerhong $M, X u Y$, Yin X-B. Review on carbon dots and their applications. Chin J Anal Chem. 2017:45(1):139-50.

66. Sagbas S, Sahiner N. Carbon dots: preparation, properties, and application. Nanocarbon and its Composites. 2019:651-76.

67. Miao P, Han K, Tang Y, Wang B, Lin T, Cheng W. Recent advances in carbon nanodots: synthesis, properties and biomedical applications. Nanoscale. 2015;7(5):1586-95.

68. Wang Y, Hu A. Carbon quantum dots: synthesis, properties and applications. J Mater Chem C. 2014:2(34):6921-39.

69. Yuan F, Li S, Fan Z, Meng X, Fan L, Yang S. Shining carbon dots: synthesis and biomedical and optoelectronic applications. Nano Today. 2016;11(5):565-86.
70. Namdari P, Negahdari B, Eatemadi A. Synthesis, properties and biomedical applications of carbon-based quantum dots: an updated review. Biomed Pharmacother. 2017;87:209-22.

71. Xie R, Wang Z, Zhou W, Liu Y, Fan L, Li Y, Li X. Graphene quantum dots as smart probes for biosensing. Anal Methods. 2016:8(20):4001-16.

72. Zhu S, Meng Q, Wang L, Zhang J, Song Y, Jin H, Zhang K, Sun H, Wang $\mathrm{H}$, Yang B. Highly photoluminescent carbon dots for multicolor patterning, sensors, and bioimaging. Angew Chem Int Ed Engl. 2013;52(14):3953-7.

73. Zhang W, Wu B, Li Z, Wang Y, Zhou J, Li Y. Carbon quantum dots as fluorescence sensors for label-free detection of folic acid in biological samples. Spectrochim Acta A Mol Biomol Spectrosc. 2020:229:117931.

74. Zheng Y, Zheng J, Wang J, Yang Y, Lu T, Liu X. Facile Preparation of stable solid-state carbon quantum dots with multi-peak emission. Nanomaterials. 2020;10(2):303.

75. Choi Y, Jo S, Chae A, Kim YK, Park JE, Lim D, Park SY, In I. Simple microwave-assisted synthesis of amphiphilic carbon quantum dots from A3/B2 polyamidation monomer set. ACS Appl Mater Interfaces. 2017;9(33):27883-93.

76. Guo Y, Wang Z, Shao H, Jiang X. Hydrothermal synthesis of highly fluorescent carbon nanoparticles from sodium citrate and their use for the detection of mercury ions. Carbon. 2013;52:583-9.

77. Li W, Wang S, Li Y, Ma C, Huang Z, Wang C, Li J, Chen Z, Liu S. Onestep hydrothermal synthesis of fluorescent nanocrystalline cellulose/ carbon dot hydrogels. Carbohydr Polym. 2017;175:7-17.

78. Wang N, Wang Y, Guo T, Yang T, Chen M, Wang J. Green preparation of carbon dots with papaya as carbon source for effective fluorescent sensing of Iron (III) and Escherichia coli. Biosens Bioelectron. 2016;85:68-75.

79. Li Z, Ni Y, Kokot S. A new fluorescent nitrogen-doped carbon dot system modified by the fluorophore-labeled ssDNA for the analysis of 6-mercaptopurine and $\mathrm{Hg}$ (II). Biosens Bioelectron. 2015;74:91-7.

80. Liu Y, Zhao Y, Zhang Y. One-step green synthesized fluorescent carbon nanodots from bamboo leaves for copper(II) ion detection. Sens Actuators, B Chem. 2014;196:647-52.

81. Li L, Li L, Chen C-P, Cui F. Green synthesis of nitrogen-doped carbon dots from ginkgo fruits and the application in cell imaging. Inorg Chem Commun. 2017;86:227-31.

82. Zhang S, Wang Z, Pang Y, Jing Z, Li Z, Peng F, Zhao Y, Guo Y. Highly fluorescent carbon dots from coix seed for the determination of furazolidone and temperature. Spectrochim Acta A Mol Biomol Spectrosc. 2021;260:119969.

83. Surendran P, Lakshmanan A, Vinitha G, Ramalingam G, Rameshkumar P. Facile preparation of high fluorescent carbon quantum dots from orange waste peels for nonlinear optical applications. Luminescence. 2020;35(2):196-202.

84. Wang M, Shi R, Gao M, Zhang K, Deng L, Fu Q, Wang L, Gao D. Sensitivity fluorescent switching sensor for $\mathrm{Cr}(\mathrm{VI})$ and ascorbic acid detection based on orange peels-derived carbon dots modified with EDTA. Food Chem. 2020;318:126506.

85. Jiang X, Qin D, Mo G, Feng J, Yu C, Mo W, Deng B. Ginkgo leaf-based synthesis of nitrogen-doped carbon quantum dots for highly sensitive detection of salazosulfapyridine in mouse plasma. J Pharm Biomed Anal. 2019;164:514-9.

86. Chen W, Yao S, Wan J, Tian Y, Huang L, Wang S, Akter F, Wu Y, Yao Y, Zhang X. BBB-crossing adeno-associated virus vector: An excellent gene delivery tool for CNS disease treatment. J Control Release. 2021;333:129-38.

87. Wu D, Huang $X$, Deng $X$, Wang $K$, Liu Q. Preparation of photoluminescent carbon nanodots by traditional Chinese medicine and application as a probe for Hg2+. Anal Methods. 2013;5(12):3023.

88. Sun D, Liu T, Wang C, Yang L, Yang S, Zhuo K. Hydrothermal synthesis of fluorescent carbon dots from gardenia fruit for sensitive on-off-on detection of $\mathrm{Hg}(2+)$ and cysteine. Spectrochim Acta A Mol Biomol Spectrosc. 2020;240:118598.

89. Bhaskar S, Tian F, Stoeger T, Kreyling W, De La Fuente JM, Grazú V, Borm P, Estrada G, Ntziachristos V, Razansky D. Multifunctional nanocarriers for diagnostics, drug delivery and targeted treatment across blood-brain barrier: perspectives on tracking and neuroimaging. Part Fibre Toxicol. 2010;7(1):3. 
90. Zhou Y, Peng Z, Seven ES, Leblanc RM. Crossing the blood-brain barrier with nanoparticles. J Control Release. 2018;270:290-303.

91. Li Y, Liu Y, Cui J, Zhao H, Liu Y, Huang L. Cohort studies on chronic noncommunicable diseases treated with traditional Chinese medicine: a bibliometric analysis. Front Pharmacol. 2021;12:639860.

92. Kang C, Huang Y, Yang H, Yan XF, Chen ZP. A review of carbon dots produced from biomass wastes. Nanomaterials. 2020;10(11):2316.

93. Wang R, Lu K-Q, Tang Z-R, Xu Y-J. Recent progress in carbon quantum dots: synthesis, properties and applications in photocatalysis. J Mater Chem A. 2017;5(8):3717-34.

94. Dager A, Uchida T, Maekawa T, Tachibana M. Synthesis and characterization of mono-disperse carbon quantum dots from fennel seeds: photoluminescence analysis using machine learning. Sci Rep. 2019;9(1):14004

95. Parvin N, Mandal TK. Synthesis of a highly fluorescence nitrogen-doped carbon quantum dots bioimaging probe and its in vivo clearance and printing applications. RSC Adv. 2016;6(22):18134-40.

96. Xue M, Zou M, Zhao J, Zhan Z, Zhao S. Green preparation of fluorescent carbon dots from lychee seeds and their application for the selective detection of methylene blue and imaging in living cells. J Mater Chem B. 2015;3(33):6783-9.

97. Zhang M, Zhao Y, Cheng J, Liu X, Wang Y, Yan X, Zhang Y, Lu F, Wang Q, Qu H. Novel carbon dots derived from Schizonepetae Herba Carbonisata and investigation of their haemostatic efficacy. Artif Cells Nanomed Biotechnol. 2018;46(8):1562-71.

98. Sun Z, Lu F, Cheng J, Zhang M, Zhang Y, Xiong W, Zhao Y, Qu H. Haemostatic bioactivity of novel Schizonepetae Spica Carbonisata-derived carbon dots via platelet counts elevation. Artif Cells Nanomed Biotechnol. 2018:46(sup3):S308-17.

99. Ghosal K, Ghosh A. Carbon dots: The next generation platform for biomedical applications. Mater Sci Eng C Mater Biol Appl. 2019:96:887-903.

100. Liu H, He Z, Jiang LP, Zhu JJ. Microwave-assisted synthesis of wavelength-tunable photoluminescent carbon nanodots and their potential applications. ACS Appl Mater Interfaces. 2015;7(8):4913-20.

101. Shen Z, Zhang C, Yu X, Li J, Wang Z, Zhang Z, Liu B. Microwave-assisted synthesis of cyclen functional carbon dots to construct a ratiometric fluorescent probe for tetracycline detection. J Mater Chem C. 2018:6(36):9636-41.

102. Chung S, Revia RA, Zhang M. Graphene quantum dots and their applications in bioimaging, biosensing, and therapy. Adv Mater. 2021;33(22):e1904362.

103. Hu X, Li Y, Xu Y, Gan Z, Zou X, Shi J, Huang X, Li Z, Li Y. Green one-step synthesis of carbon quantum dots from orange peel for fluorescent detection of Escherichia coli in milk. Food Chem. 2021;339:127775.

104. Agrawal A, Cho SH, Zandi O, Ghosh S, Johns RW, Milliron DJ. Localized surface plasmon resonance in semiconductor nanocrystals. Chem Rev. 2018;118(6):3121-207.

105. Bawarski WE, Chidlowsky E, Bharali DJ, Mousa SA. Emerging nanopharmaceuticals. Nanomedicine. 2008:4(4):273-82.

106. Pautler M, Brenner S. Nanomedicine: promises and challenges for the future of public health. Int J Nanomed. 2010:5:803-9.

107. Mansur HS, Mansur AAP, Curti E, De Almeida MV. Functionalizedchitosan/quantum dot nano-hybrids for nanomedicine applications: towards biolabeling and biosorbing phosphate metabolites. J Mater Chem B. 2013;1 (12):1696-711.

108. Chen Z, Ye SY, Yang Y, Li ZY. A review on charred traditional Chinese herbs: carbonization to yield a haemostatic effect. Pharm Biol. 2019;57(1):498-506

109. Yan X, Zhao Y, Luo J, Xiong W, Liu X, Cheng J, Wang Y, Zhang M, Qu H. Hemostatic bioactivity of novel pollen typhae carbonisata-derived carbon quantum dots. J Nanobiotechnol. 2017;15(1):60.

110. Liu X, Wang Y, Yan X, Zhang M, Zhang Y, Cheng J, Lu F, Qu H, Wang Q, Zhao Y. Novel Phellodendri Cortex (Huang Bo)-derived carbon dots and their hemostatic effect. Nanomedicine. 2018:13(4):391-405.

111. Drayton DL, Liao S, Mounzer RH, Ruddle NH. Lymphoid organ development: from ontogeny to neogenesis. Nat Immunol. 2006;7(4):344-53.

112. Karin $M$, Lawrence T, Nizet $V$. Innate immunity gone awry: linking microbial infections to chronic inflammation and cancer. Cell. 2006;124(4):823-35.
113. Tasneem S, Liu B, Li B, Choudhary MI, Wang W. Molecular pharmacology of inflammation: medicinal plants as anti-inflammatory agents. Pharmacol Res. 2019:139:126-40.

114. Huang Y, Cai T, Xia X, Cai Y, Wu XY. Research advances in the intervention of inflammation and cancer by active ingredients of traditional Chinese medicine. J Pharm Pharm Sci. 2016;19(1):114.

115. Hu J, Luo J, Zhang M, Wu J, Zhang Y, Kong H, Qu H, Cheng G, Zhao Y. Protective effects of Radix Sophorae Flavescentis carbonisatabased carbon dots against ethanol-induced acute gastric ulcer in rats: anti-inflammatory and antioxidant activities. Int J Nanomed. 2021;16:2461-75

116. Wu J, Zhang M, Cheng J, Zhang Y, Luo J, Liu Y, Kong H, Qu H, Zhao Y. Effect of Lonicerae japonicae Flos carbonisata-derived carbon dots on rat models of fever and hypothermia induced by lipopolysaccharide. Int J Nanomed. 2020;15:4139-49.

117. Wang S, Zhang Y, Kong H, Zhang M, Cheng J, Wang X, Lu X, Qu H, Zhao Y. Antihyperuricemic and anti-gouty arthritis activities of Aurantii fructus immaturus carbonisata-derived carbon dots. Nanomedicine. 2019;14(22):2925-39.

118. Wang $X$, Zhang Y, Zhang M, Kong H, Wang S, Cheng J, Qu H, Zhao Y. Novel carbon dots derived from Puerariae lobatae Radix and their antigout effects. Molecules. 2019;24(22):4152.

119. Dalbeth N, Merriman TR, Stamp LK. Gout. Lancet. 2016;388(10055):2039-52

120. Zhang ZC, Zhou Q, Yang Y, Wang Y, Zhang JL. Highly acylated anthocyanins from purple sweet potato (Ipomoea batatas L.) alleviate hyperuricemia and kidney inflammation in hyperuricemic mice: possible attenuation effects on allopurinol. J Agric Food Chem. 2019:67(22):6202-11.

121. Seth R, Kydd AS, Buchbinder R, Bombardier C, Edwards CJ. Allopurinol for chronic gout. Cochrane Database Syst Rev. 2014;10:CD006077.

122. Quach C, Galen BT. HLA-B*5801 testing to prevent allopurinol hypersensitivity syndrome: a teachable moment. JAMA Intern Med. 2018;178(9):1260-1

123. Stamp LK, Barclay ML. How to prevent allopurinol hypersensitivity reactions? Rheumatology. 2018;57(suppl_1):i35-41.

124. Wang CW, Dao RL, Chung WH. Immunopathogenesis and risk factors for allopurinol severe cutaneous adverse reactions. Curr Opin Allergy Clin Immunol. 2016;16(4):339-45.

125. He J. The potential contributions of traditional Chinese medicine to emergency medicine. World J Emerg Med. 2013;4(2):92.

126. Zhao Y, Zhang Y, Kong H, Zhang M, Cheng J, Wu J, Ou H, Zhao Y. Carbon dots from Paeoniae Radix Alba Carbonisata: hepatoprotective effect. Int J Nanomed. 2020;15:9049-59.

127. Zhang $M$, Cheng J, Sun Z, Kong $H$, Zhang $Y$, Wang S, Wang $X$, Zhao Y, Qu $\mathrm{H}$. Protective effects of carbon dots derived from Phellodendri Chinensis Cortex carbonisata against deinagkistrodon acutus venom-induced acute kidney injury. Nanoscale Res Lett. 2019;14(1):377.

128. Liu Y, Yang S, Wang K, Lu J, Bao X, Wang R, Qiu Y, Wang T, Yu H. Cellular senescence and cancer: focusing on traditional Chinese medicine and natural products. Cell Prolif. 2020;53(10):e12894.

129. Xiang Y, Guo Z, Zhu P, Chen J, Huang Y. Traditional Chinese medicine as a cancer treatment: modern perspectives of ancient but advanced science. Cancer Med. 2019;8(5):1958-75.

130. So TH, Chan SK, Lee VH, Chen BZ, Kong FM, Lao LX. Chinese medicine in cancer treatment-how is it practised in the East and the West? Clin Oncol. 2019;31(8):578-88.

131. Kim W, Egan JM. The role of incretins in glucose homeostasis and diabetes treatment. Pharmacol Rev. 2008;60(4):470-512.

132. Sun Z, Lu F, Cheng J, Zhang M, Zhu Y, Zhang Y, Kong H, Qu H, Zhao Y. Hypoglycemic bioactivity of novel eco-friendly carbon dots derived from traditional Chinese medicine. J Biomed Nanotechnol. 2018;14(12):2146-55.

133. Lorentzen AK, Davis C, Penninga L. Interventions for frostbite injuries. Cochrane Database Syst Rev. 2018. https://doi.org/10.1002/14651858. CD012980.pub2.

134. Kong H, Zhao Y, Zhu Y, Xiong W, Luo J, Cheng J, Zhang Y, Zhang M, Qu H, Zhao Y. Carbon dots from Artemisiae Argyi Folium Carbonisata: strengthening the anti-frostbite ability. Artif Cells Nanomed Biotechnol. 2021:49(1):11-9. 
135. Zhang M, Cheng J, Zhang Y, Kong H, Wang S, Luo J, Qu H, Zhao Y. Green synthesis of Zingiberis rhizoma-based carbon dots attenuates chemical and thermal stimulus pain in mice. Nanomedicine. 2020;15(9):851-69.

136. Servick K. Safety benefits of "biased" opioids scrutinized. Science. 2020;367(6481):966

137. Ariya PA, Amyot M, Dastoor A, Deeds D, Feinberg A, Kos G, Poulain A, Ryjkov A, Semeniuk K, Subir M, Toyota K. Mercury physicochemical and biogeochemical transformation in the atmosphere and at atmospheric interfaces: a review and future directions. Chem Rev. 2015;115(10):3760-802.

138. Okonko DO, Mandal AKJ, Missouris CG, Poole-Wilson PA. Disordered iron homeostasis in chronic heart failure. J Am Coll Cardiol. 2011;58(12):1241-51.

139. Brucker D, Leopold K. Sizing silver nanoparticles in chicken meat using direct slurry sampling graphite furnace atomic absorption spectrometry. Anal Bioanal Chem. 2019;411(19):4551-8.

140. Bulska E, Wagner B. Quantitative aspects of inductively coupled plasma mass spectrometry. Philos Trans Royal Soc A. 2016;374(2079):20150369.

141. Zhang Z, Ji H, Song Y, Zhang S, Wang M, Jia C, Tian J-Y, He L, Zhang $X$, Liu C-S. Fe(III)-based metal-organic framework-derived core-shell nanostructure: sensitive electrochemical platform for high trace determination of heavy metal ions. Biosens Bioelectron. 2017;94:358-64.

142. Zhao X, Liao S, Wang L, Liu Q, Chen X. Facile green and one-pot synthesis of Purple perilla derived carbon quantum dot as a fluorescent sensor for silver ion. Talanta. 2019;201:1-8.

143. Barati A, Shamsipur M, Abdollahi H. Hemoglobin detection using carbon dots as a fluorescence probe. Biosens Bioelectron. 2015:71:470-5.

144. Hu Y, Gao Z, Luo J. Fluorescence detection of malachite green in fish tissue using red emissive $\mathrm{Se}, \mathrm{N} \mathrm{Cl-doped} \mathrm{carbon} \mathrm{dots.} \mathrm{Food} \mathrm{Chem.}$ 2021;335:127677.

145. Pu J, Liu C, Wang B, Liu P, Jin Y, Chen J. Orange red-emitting carbon dots for enhanced colorimetric detection of Fe(3). Analyst. 2021;146(3):1032-9.

146. Song Y, Zhu S, Xiang S, Zhao X, Zhang J, Zhang H, Fu Y, Yang B. Investigation into the fluorescence quenching behaviors and applications of carbon dots. Nanoscale. 2014;6(9):4676-82.

147. Chang D, Shi L, Zhang Y, Zhang G, Zhang C, Dong C, Shuang S. Smilax China-derived yellow-fluorescent carbon dots for temperature sensing, $\mathrm{Cu}(2+)$ detection and cell imaging. Analyst. 2020;145(6):2176-83.

148. Sun X, He J, Yang S, Zheng M, Wang Y, Ma S, Zheng H. Green synthesis of carbon dots originated from Lycii Fructus for effective fluorescent sensing of ferric ion and multicolor cell imaging. J Photochem Photobiol B. 2017;175:219-25.

149. Kim K, Kim J. Synthesis of carbon quantum dots from jujubes for detection of Iron(III) ions. J Nanosci Nanotechnol. 2018;18(2):1320-2.

150. Wang M, Wan Y, Zhang K, Fu Q, Wang L, Zeng J, Xia Z, Gao D. Green synthesis of carbon dots using the flowers of Osmanthus fragrans (Thunb.)
Lour. as precursors: application in Fe(3+) and ascorbic acid determination and cell imaging. Anal Bioanal Chem. 2019;411(12):2715-27.

151. Dai J. Nitrogen-doped carbon quantum dots with Pinellia ternata as carbon source for high sensitive determination of chromium (Vi). Appl Ecol Environ Res. 2019:17(5):12139-53.

152. Yu C, Qin D, Jiang X, Zheng X, Deng B. N-doped carbon quantum dots from Osmanthus fragrans as a novel off-on fluorescent nanosensor for highly sensitive detection of quercetin and aluminium ion, and cell imaging. J Pharm Biomed Anal. 2021;192:113673.

153. Feng $Y$, Zhong D, Miao H, Yang X. Carbon dots derived from rose flowers for tetracycline sensing. Talanta. 2015;140:128-33.

154. Sharma V, Tiwari P, Mobin SM. Sustainable carbon-dots: recent advances in green carbon dots for sensing and bioimaging. J Mater Chem B. 2017;5(45):8904-24

155. He M, Zhang J, Wang H, Kong Y, Xiao Y, Xu W. Material and optical properties of fluorescent carbon quantum dots fabricated from lemon juice via hydrothermal reaction. Nanoscale Res Lett. 2018;13(1):175.

156. Lu Y, Aimetti AA, Langer R, Gu Z. Bioresponsive materials. Nat Rev Mater. 2016;2(1):1-7

157. Chandra S, Singh VK, Yadav PK, Bano D, Kumar V, Pandey VK, Talat M, Hasan SH. Mustard seeds derived fluorescent carbon quantum dots and their peroxidase-like activity for colorimetric detection of $\mathrm{H} 2 \mathrm{O} 2$ and ascorbic acid in a real sample. Anal Chim Acta. 2019;1054:145-56.

158. Shi X, Meng H, Sun Y, Qu L, Lin Y, Li Z, Du D. Far-red to near-infrared carbon dots: preparation and applications in biotechnology. Small. 2019;15(48):e1901507.

159. Pearce $S$, Nezich CL, Spinazzola A. Mitochondrial diseases: translation matters. Mol Cell Neurosci. 2013;55:1-12.

160. Zhou Y, Selvam A, Wong JW. Effect of Chinese medicinal herbal residues on microbial community succession and anti-pathogenic properties during co-composting with food waste. Bioresour Technol. 2016;217:190-9.

161. Wei X, Li L, Liu J, Yu L, Li H, Cheng F, Yi X, He J, Li B. Green Synthesis of fluorescent carbon dots from gynostemma for bioimaging and antioxidant in zebrafish. ACS Appl Mater Interfaces. 2019;11(10):9832-40.

162. Zhang M, Cheng J, Hu J, Luo J, Zhang Y, Lu F, Kong H, Qu H, Zhao Y. Green Phellodendri Chinensis Cortex-based carbon dots for ameliorating imiquimod-induced psoriasis-like inflammation in mice. J Nanobiotechnol. 2021;19(1):105.

163. Cheng J, Zhang M, Sun Z, Lu F, Xiong W, Luo J, Kong H, Wang Q, Qu H, Zhao Y. Hemostatic and hepatoprotective bioactivity of Junci Medulla Carbonisata-derived carbon dots. Nanomedicine. 2019;14(4):431-46.

\section{Publisher's Note}

Springer Nature remains neutral with regard to jurisdictional claims in published maps and institutional affiliations.
Ready to submit your research? Choose BMC and benefit from:

- fast, convenient online submission

- thorough peer review by experienced researchers in your field

- rapid publication on acceptance

- support for research data, including large and complex data types

- gold Open Access which fosters wider collaboration and increased citations

- maximum visibility for your research: over 100M website views per year

At BMC, research is always in progress.

Learn more biomedcentral.com/submissions 